\title{
基于糖烯的碳苷合成方法研究进展
}

\author{
郭真言柏金和刘苗熊德彩* 叶新山*
}

(北京大学天然药物及仿生药物国家重点实验室 北京 100191)

\begin{abstract}
摘要 碳苷是一类具有多种生物活性的糖类天然产物, 其独特的 $\mathrm{C}-\mathrm{C}$ 糖苷键构筑是糖化学研究中的热点和难点. 通 过双键官能团构建新的 $\mathrm{C}-\mathrm{C}$ 键是有机化学中的常用策略. 随着烯化学的飞速发展, 含 1,2-位双键的糖烯供体在碳苷合 成中也取得了显著进步. 从 Ferrier I 型碳苷化反应、Heck 偶联型碳苷化反应、1-取代糖烯的过渡金属催化偶联碳苷化 反应、2-取代糖烯的 Michael 加成型和自由基加成型碳苷化反应等方面，总结近年来基于糖烯的碳苷合成方法.

关键词 糖烯; 碳苷; 碳苷化反应; 碳水化合物
\end{abstract}

\section{Advances in the Synthesis of C-Glycosides from Glycals}

\author{
Guo, Zhenyan Bai, Jinhe Liu, Miao Xiong, Decai* Ye, Xinshan* \\ (State Key Laboratory of Natural and Biomimetic Drugs, School of Pharmaceutical Sciences, \\ Peking University, Beijing 100191)
}

\begin{abstract}
C$-Glycosides are a class of carbohydrates with a variety of biological activities, and the construction of their unique $\mathrm{C}-\mathrm{C}$ glycosidic bond is a hot and challenging topic in carbohydrate chemistry. The formation of new $\mathrm{C}-\mathrm{C}$ bond from carbon-carbon double bond is a common strategy in organic chemistry. With the rapid development of ene-chemistry, great progress in the synthesis of $C$-glycosides from glycal donors containing 1,2-double bond has been made. In this review, the methods for the synthesis of $C$-glycosides based on glycals are summarized, including Ferrier I-type $C$-glycosylation, Heck-type $C$-glycosylation, $C$-glycosylation of 1-substituted glycals using transition metal-catalyzed coupling reaction, Michael-type $C$-glycosylation of 2-substituted glycals, and radical addition type $C$-glycosylation of glycals.

Keywords glycal; $C$-glycoside; $C$-glycosylation; carbohydrate
\end{abstract}

碳苷是指糖上端基碳原子和配糖体通过碳一碳键相 连的糖类化合物. 自然界存在的天然碳苷化合物多具有 重要的生理活性。假尿嘧啶核苷 ${ }^{[1]}$ (pseudouridine) (Scheme 1) 是第一个被报道的碳核苷. 它被认为是 tRNA 降解的最终产物, 在肿瘤患者体内含量会异常增高, 可 以作为肿瘤标志物. 从中药葛根中分离得到的异黄酮类 碳苷葛根素 ${ }^{[2]}$ (puerarin), 具有舒张血管、缓解心绞痛等 多种活性. 很多具有重要生理活性的天然产物也含有碳 苷单元, 比如 Kendomycin ${ }^{[3]}$. 与氧苷的 $\mathrm{C}-\mathrm{O}$ 键相比, 碳苷端基的 $\mathrm{C}-\mathrm{C}$ 键对酶和化学(酸)水解有更强的耐受 性. 例如, $\alpha$-半乳糖神经酰胺 KRN7000 的碳苷类似物对 小鼠 B16 黑色素瘤细胞的保护作用比其氧苷母体 KRN7000 强 1000 倍 ${ }^{[4]}$; 由根皮苷(phlorizin)改造后的碳
苷类似物可以用于糖尿病的治疗, 已有多个药物品种成 功上市, 如达格列净(Dapagliflozin). 由于碳苷良好的药 用价值, 碳苷的合成研究越来越被人们所重视. 但碳碳键的构筑却是一项富有挑战性的工作. 迄今为止, 已 经有许多关于碳苷合成方法和策略的报道 ${ }^{[5]}$. 本文主要 总结基于糖烯的碳苷合成方法.

糖烯通常是指糖环的 1,2 位含有双键的糖类化合物. 对双键进行官能团化从而构筑新的碳一碳键是有机化学 中常用的策略. 糖烯具有与烯基醚和烯丙醇相似的烯 键; 理论上, 烯基醚和烯丙醇底物能发生的碳一碳键构 筑反应均可用于碳苷的合成. 糖烯双键取代基的不同, 其构筑碳一碳糖苷键的方式也不一样. 本文将按照反应 类型，从 Ferrier I 型碳苷化反应、Heck 偶联型碳苷化反

\footnotetext{
* Corresponding authors. E-mail: xinshan@bjmu.edu.cn; decai@bjmu.edu.cn Received June 5, 2020; revised July 2, 2020; published online July 22, 2020 Dedicated to Professor Henry N. C. Wong on the occasion of his 70th birthday. Project supported by the National Key Research and Development Program of China (No. 2018YFA0507602), the National Natural Science Foundation of China (No. 21738001), and the National New Drug Innovation Major Project of China (No. 2019ZX09301106).

国家重点研发计划(No. 2018YFA0507602)、国家自然科学基金项目(No. 21738001)、国家重大新药创制专项(No. 2019ZX09301106)资助项目.
} 
应、1-取代糖烯的过渡金属催化偶联碳苷化反应、2-取 代糖烯的 Michael 加成型和自由基加成型碳苷化反应 (Scheme 2)，来总结基于糖烯的碳苷合成方法.

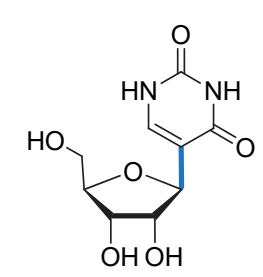

Pseudouridine<smiles>CC(=C[C@@H](C)CCC1O[C@H](C2=C(O)C(=O)C(C)=C3OC(O)(C(C)CC(C)C)C=C32)[C@H]([14CH3])[C@@H](O)[C@@H]1C)CC(C)C</smiles>

(-)-Kendomycin
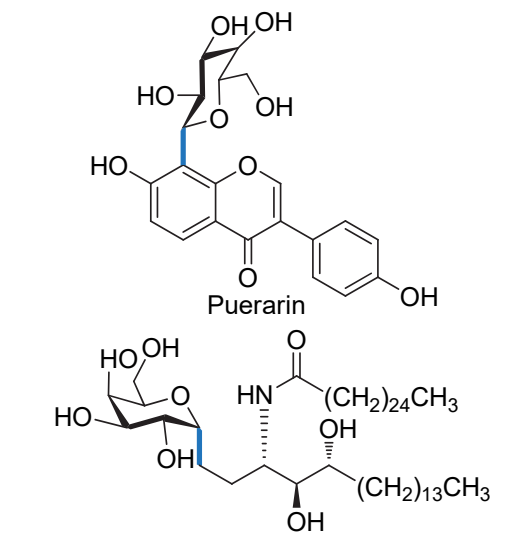

KRN7000 C-glycoside analogue

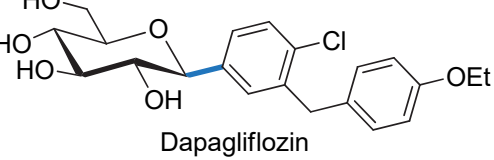

图式 1 代表性生物活性碳苷

Scheme 1 Representative bioactive $C$-glycosides<smiles>[R6]CC1OC=CC([R])C1O[R20]</smiles>

图式 2 糖烯的结构及反应

Scheme 2 Structures and reaction types of glycals

\section{Ferrier I 型碳苷化反应}

在 Lewis 酸等催化剂的作用下, 糖烯 $\mathbf{I}$ 的 3 位酰(烷) 氧基离去, 产生离域的氧杂烯丙基碳正离子 II 继而与亲 核试剂反应得到 2,3 -不饱和糖苷化合物的反应称为 Ferrier I 型反应. 由于电子效应等因素影响, 产物一般 以 $\alpha$-构型为主; 通过理论计算, 一般 $\alpha$-构型产物的半椅 式构象能量较低(Scheme 3$)^{[6]}$; 当使用含碳亲核试剂时 能得到碳苷. 根据含碳亲核试剂种类的不同, 可分为有 机硅亲核试剂、有机金属亲核试剂和不饱和烃亲核试剂 等.

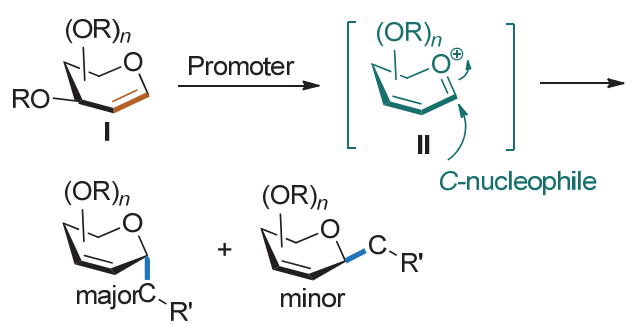

图式 3 糖烯的 Ferrier I 型碳苷化反应

Scheme 3 Ferrier I type $C$-glycosylation of glycals

\section{1 有机硅作为亲核试剂的 Ferrier I 型反应}

有机硅试剂是一种理想的亲核试剂. 利用有机硅亲 核试剂和糖烯通过 Ferrier I 型反应得到以 $\alpha$ 构型为主的 2,3-不饱和碳苷. 有机硅试剂包括烯丙基硅、炔丙基硅 以及烯醇硅醚等. 根据使用 Lewis 酸的不同, 底物适用 性和选择性略有差异.

2001 年, Schmidt 课题组 ${ }^{[7 \mathrm{a}]}$ 以 $\mathrm{Yb}(\mathrm{OTf})_{3}$ 为 Lewis 酸, 乙酰基保护的葡萄糖烯为糖基供体，烯丙基三甲基硅 基/炔丙基三甲基硅基/烯醇三甲基硅醚为亲核试剂, 在 二氯甲烷中进行反应得到不同的 2,3-不饱和烷基碳苷, 产物以 $\alpha$ 构型为主(Scheme 4). Grée 课题组 ${ }^{[7 b]}$ 发现该反 应在离子液中也可以很好地进行.
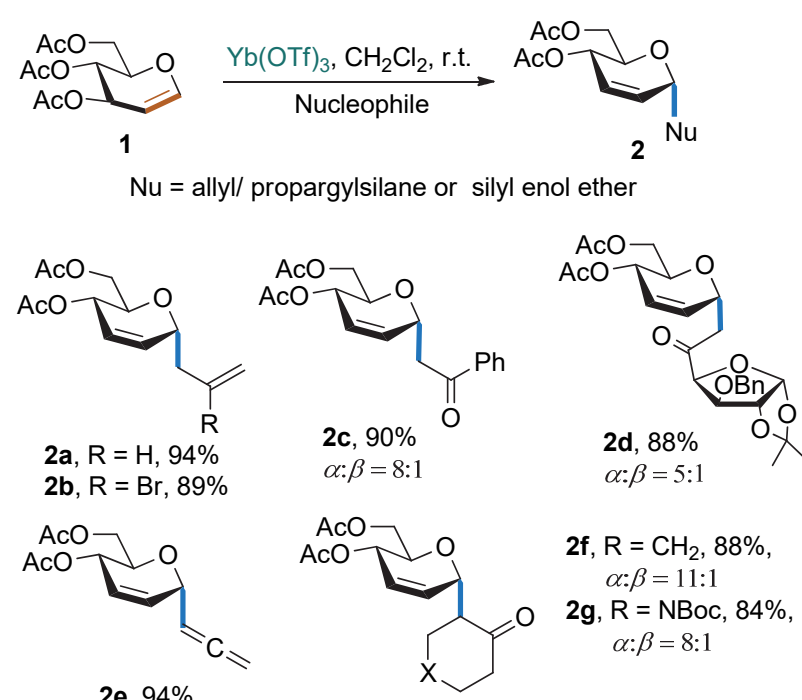

2e, $94 \%$

图式 $4 \mathrm{Yb}(\mathrm{OTf})_{3}$ 促进的碳苷合成

Scheme $4 \quad \mathrm{Yb}(\mathrm{OTf})_{3}$ catalyzed synthesis of $C$-glycosides

分子碘也可以作为催化剂使糖烯与有机硅试剂发 生碳苷化反应(Scheme 5). Isobe 课题组 ${ }^{[8 a]}$ 发现, 在 $I_{2}$ 的 作用下全乙酰化的葡萄糖烯可以与不同取代的炔基三 甲基硅烷发生 Ferrier I 型碳苷化反应，得到全 $\alpha$-构型的 炔苷. Yadav 课题组 ${ }^{[8 b]}$ 也通过碘分子完成了多种糖烯的 烯丙基化和㲵基化反应; 该反应只能得到以 $\alpha$-构型为主 的混合物.

Sachwani 课题组 ${ }^{[9]}$ 利用 $\mathrm{InCl}_{3}$ 作为 Lewis 酸, 在微波 的作用下实现了糖烯与有机硅烷的碳苷化反应(Scheme 6). 该反应对亲核试剂有限制，必须是烯丙基三甲基硅 和氰基三甲基硅，生成的碳苷以 $\alpha$ 构型为主，并不能保 证为单一构型.

2014 年, Vankar 课题组 ${ }^{[10]}$ 用硝酸铈铵 (CAN)作为氧 化剂, 以单电子氧化的方式使糖烯转化成离域的氧杂烯 丙基碳正离子，然后与烯丙基三甲基硅和氰基三甲基硅 在乙腈中进行反应, 得到碳苷(Scheme 7). 该方法适用 于不同保护基的多种糖烯，但亲核试剂只能是烯丙基三 


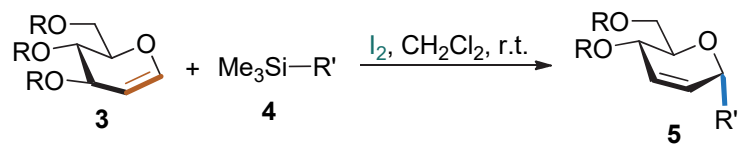

$\mathrm{R}=$ acetyl benzoyl and pivaloyl; $\mathrm{R}^{\prime}$ = allyl, alkynyl, cyanide and azide

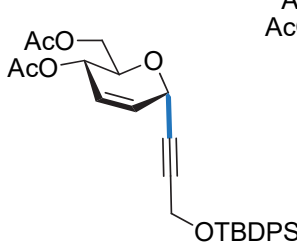

5a, $74 \%$

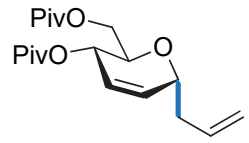

5d, $92 \%, \alpha: \beta=9: 1$

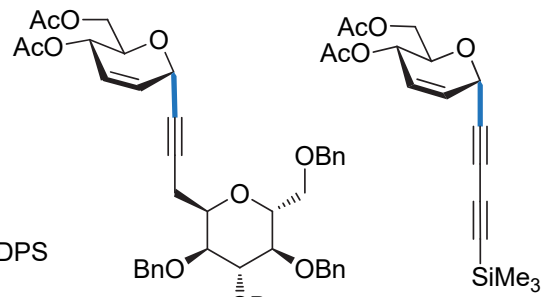

$5 b, 88 \%$

5c, $67 \%$

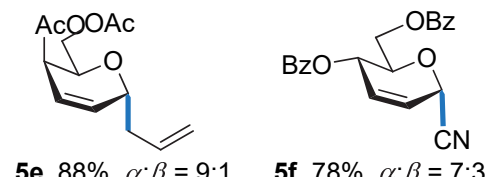

图式 $5 \mathrm{I}_{2}$ 促进的碳苷合成

Scheme $5 \quad \mathrm{I}_{2}$ catalyzed synthesis of $C$-glycosides

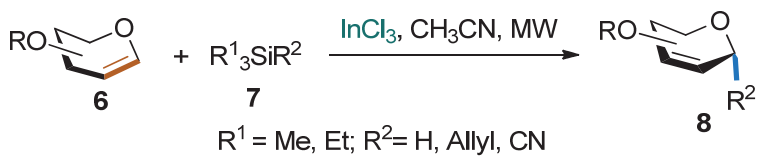<smiles></smiles>

8a, $85 \%$ $\alpha: \beta=13: 1$

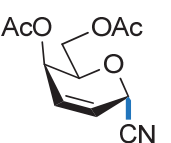

$\mathbf{8 b}, 79 \%$ $\alpha: \beta=3: 2$

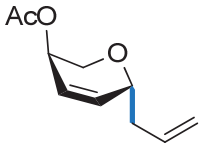

8c, $89 \%$ $\alpha: \beta=19: 1$

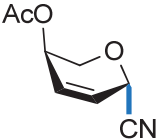

$8 d, 72 \%$ $\alpha: \beta=10: 1$
图式 $6 \mathrm{InCl}_{3}$ 促进的碳苷合成

Scheme $6 \mathrm{InCl}_{3}$ catalyzed synthesis of $C$-glycosides

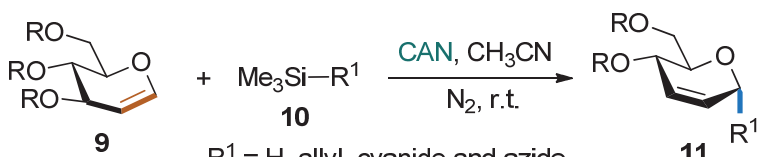
$\mathrm{R}^{1}=\mathrm{H}$, allyl, cyanide and azide $11^{R}$

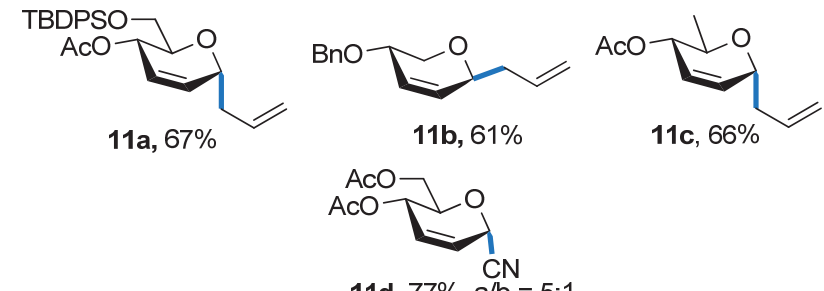

图式 $7 \mathrm{CAN}$ 介导的碳苷合成

Scheme 7 CAN promoted synthesis of $C$-glycosides

甲基硅和氧基三甲基硅. 作者利用该方法进行了 2-脱 氧-2-氨基碳苷的合成.

\section{2 有机锌/锂/铝作为亲核试剂的 Ferrier I 型反应}

2001 年, Bois 课题组 ${ }^{[1]]}$ 在乙醚为溶剂和室温条件 下, 实现了有机锌试剂参与的 Ferrier I 型反应, 得到 $\alpha$ 构型为主的碳苷(Scheme 8a). 该反应具有良好的底物适
用性，能同时适用于脂肪族和芳香族的有机锌试剂. 有 机锌化合物可由卤代烃在叔丁醇锂和氯化锌的存在下 原位制得，并直接用于碳苷合成(Scheme 8b).

(a)

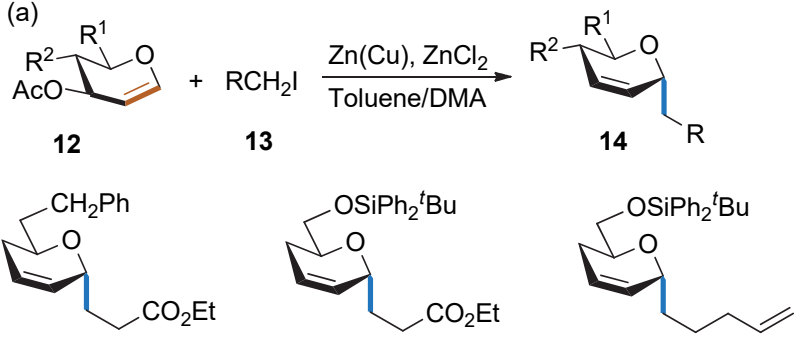

$14 a, 72 \%$

$14 b, 72 \%$

14c, $63 \%$

(b)

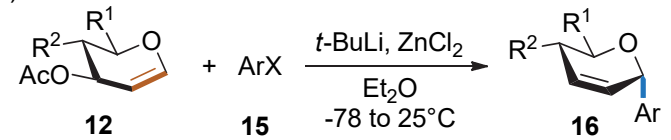

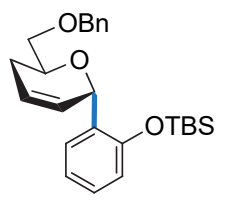

$16 a, 82 \%$

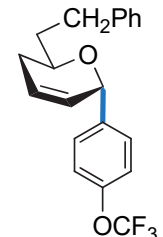

16b, $78 \%$

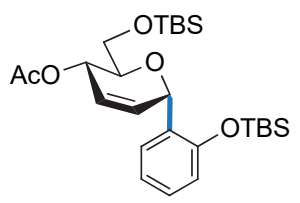

16c, $75 \%$
图式 8 烷基/芳基锌作为 Ferrier I 型反应的亲核试剂

Scheme 8 Alkylzinc/arylzinc as the nucleophile for Ferrier I reaction

2014 年, Mukherjee 课题组 ${ }^{[12]}$ 利用糖烯 17、端炔、 溴代乙酸乙酯和锌粉发展了一种新的炔基碳苷合成方 法(Scheme 9). 该方法以原位生成的炔基锌为亲核试剂, 具有良好的底物适用性和 $\alpha$ 立体选择性. 值得一提的是, 该方法还适用于 2-乙酰氧基取代的糖烯。

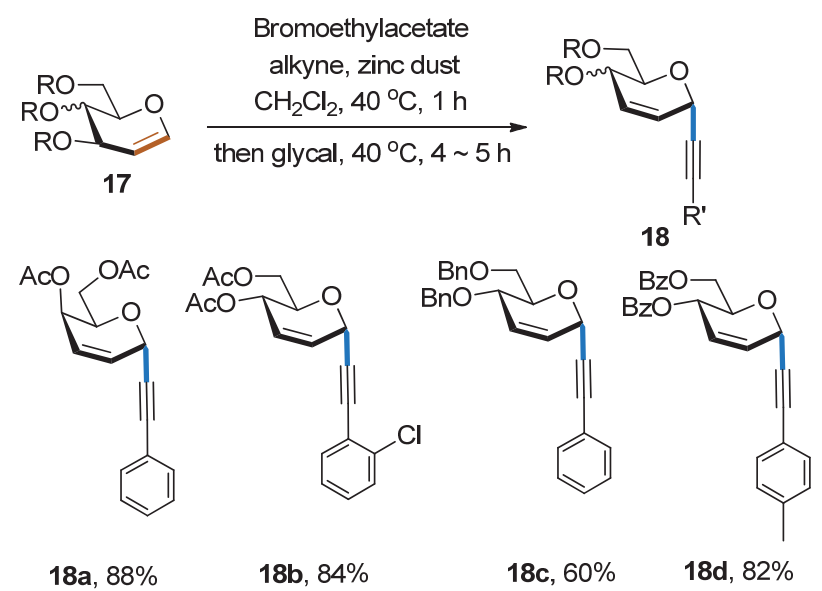

图式 9 炔基锌作为 Ferrier I 型反应的亲核试剂 Scheme 9 Alkynylzinc as the nucleophile for Ferrier I reaction 2003 年, Crotti 课题组 ${ }^{[13]}$ 发现 3-着基-4-甲磺酰氧基 
糖烯在叔丁醇钾的作用下可得到烯基环氧中间体. 该中 间体可作为供体与有机锂亲核试剂反应得到碳苷 (Scheme 10). 产物的构型与生成的环氧中间体的构型 有关, 有机锂试剂可通过与环氧协同配位, 生成与环氧 同向的产物. 简单的烷基锂和芳基锂都可顺利进行此反 应.

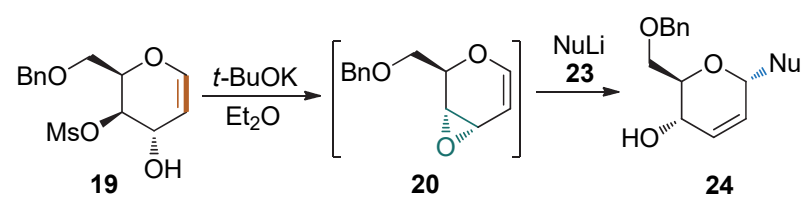

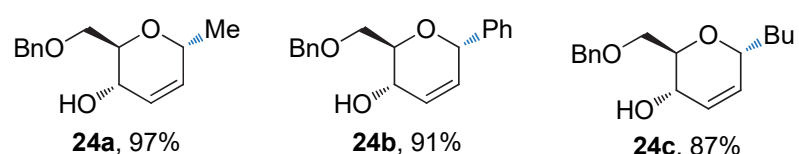

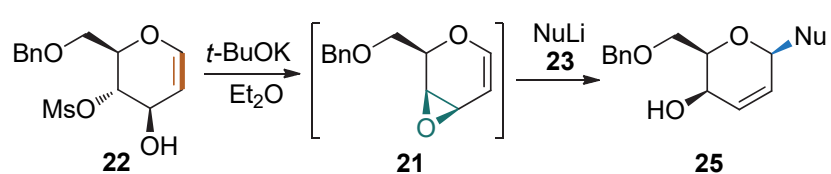<smiles>CC(C)C1C=CC(O)C(COCc2ccccc2)O1</smiles>

25a, $80 \%$

25b, $93 \%$

25c, $78 \%$

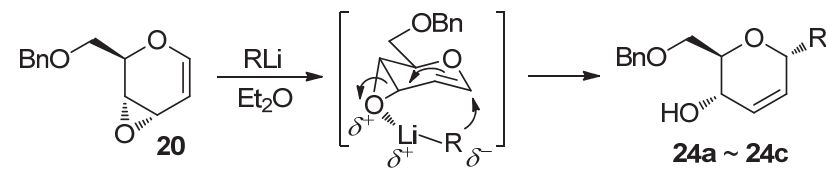

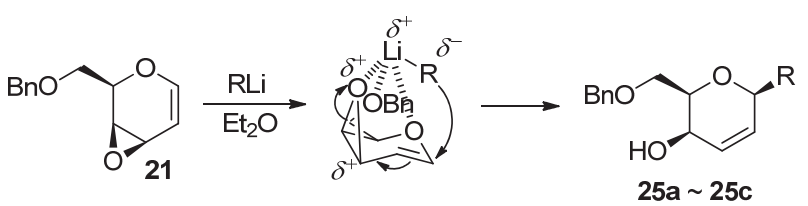

图式 10 有机锂作为 Ferrier I 型反应的亲核试剂 Scheme 10 Organolithium as the nucleophile for Ferrier I reaction

2009 年, Pohmakotr 课题组 ${ }^{[14]}$ 发现, 在 $\mathrm{Yb}(\mathrm{OTf})_{3}$ 的 作用下, 糖烯可与有机铝发生 Ferrrier 重排反应, 得到碳 苷产物(Scheme 11). 该方法对糖型和保护基有较好的耐 受性, 收率良好, 但亲核试剂只有甲基铝和乙基铝, 立 体选择性较差.

\section{3 炔铟/硼/铜/金作为亲核试剂的 Ferrier I 型反应}

炔基本身很难作为 Ferrier I 型反应的亲核试剂, 因 此, 要制备炔苷通常需要先将端炔活化. 2007 年, Auge 课题组 ${ }^{[15]}$ 发现金属铟可以原位与碘代炔反应, 生成炔 基铟, 进而与糖烯发生 Ferrier I 型反应, 生成炔基碳苷 (Scheme 12). 该方法对糖烯具有较好的底物适用性, 收 率较好, 但反应时间长, 立体选择性中等.

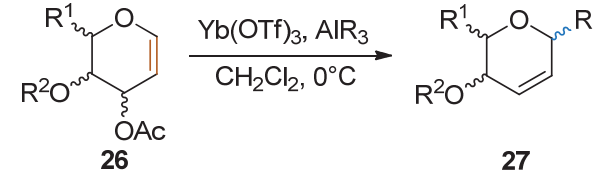

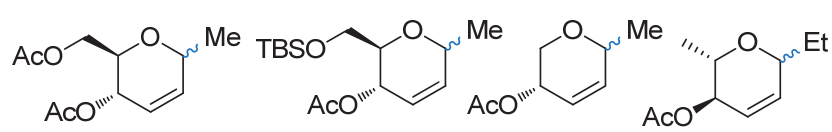

27a, $94 \%, \quad 27 b, 88 \% \quad 27 c, 81 \% \quad 27$ d, $95 \%$ $\alpha: \beta=79: 21 \quad \alpha: \beta=75: 25 \quad \alpha: \beta=85: 15 \quad \alpha: \beta=70: 30$

图式 11 三烷基铝作为 Ferrier I 型反应的亲核试剂 Scheme 11 Trialkyl aluminum as the nucleophile for Ferrier I reaction<smiles>[R]C#C[C@H]1C=C[C@@H]([OH2+])[C@@H](C[R])O1</smiles>

30a, 95\%, $\alpha / \beta$ 83:17

30b, 93\%, $\alpha / \beta$ 85:15

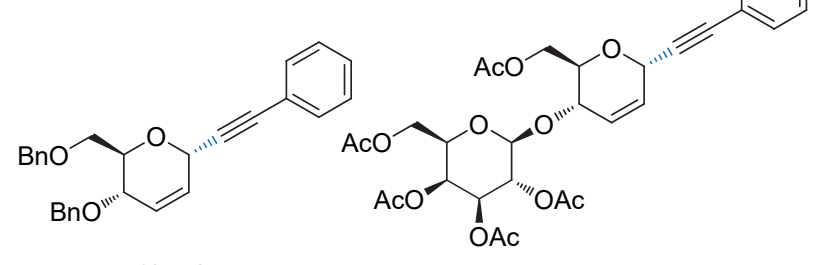

30c, $67 \%, \alpha \mid \beta>95: 5$

30d, $65 \%, \alpha / \beta 78: 22$

图式 12 炔基铟作为 Ferrier I 型反应的亲核试剂 Scheme 12 Alkynyl indium as the nucleophile for Ferrier I reaction

2008 年, Stefani 课题组 ${ }^{[16]}$ 发现糖烯可以在三氟化硼 乙醚的催化下与炔基三氟硼酸钾发生 Ferrier I 型反应, 生成炔基碳苷。该反应条件温和，在 $0{ }^{\circ} \mathrm{C}$ 条件下乙腈溶 液中 $0.5 \mathrm{~h}$ 即可反应完全. 反应对亲核试剂有良好的底 物适用范围. 产物的立体选择性好, $\alpha / \beta$ 比例大于 $98 / 7$ (Scheme 13).

Mukherjee 课题组 ${ }^{[17]}$ 在 2013 年做过炔基碳苷的合成 研究(Scheme 14). 他们发现, 在抗坏血酸的存在下, $\mathrm{Cu}(\mathrm{OTf})_{2}$ 可以作为催化剂促进糖烯与不活泼的炔烃的 快速偶联得到碳苷. 该反应经历炔铜中间体，反应收率 高、反应时间短 $(2 \mathrm{~min}$ 以内)、条件温和、 $\alpha$ 立体选择性 大于 96/4.

2016 年，史晓东课题组 ${ }^{[18]}$ 用(ArO) ${ }_{3} \mathrm{PAu}(\mathrm{TA}-\mathrm{Ph}) \mathrm{OTf}$ 作为催化剂, 通过炔金中间体进一步将该反应拓展到不 活泼糖烯同不活泼炔烃的 Ferrier I 型反应. 他们还将亲 核试剂拓展到一些含天然产物片段的芳炔的合成，进一 步展示了该反应的实用性(Scheme 15). 


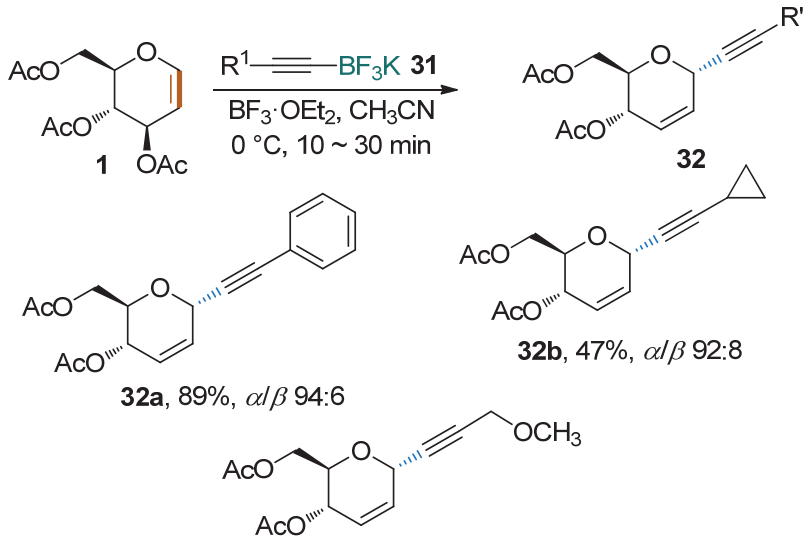

32c, $82 \%, \alpha / \beta>98: 2$

图式 13 炔基三氟硼酸钾作为 Ferrier I 型反应的亲核试剂 Scheme 13 Potassium alkynyltrifluoroborate as the nucleophile for Ferrier I reaction

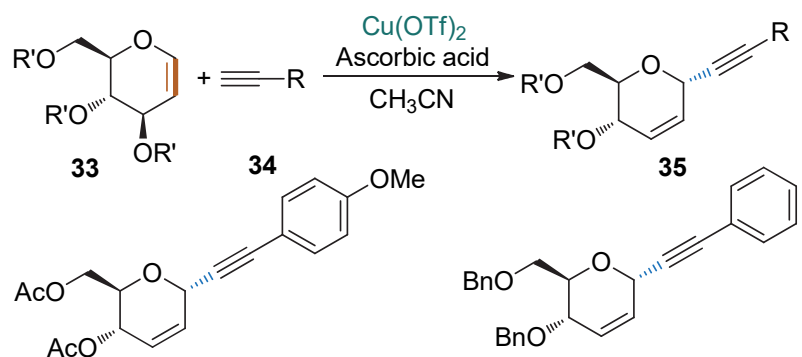<smiles>O=C(OCC1O[C@H](C#Cc2ccccc2)C=CC1OC(=O)OCc1ccccc1)Oc1ccccc1</smiles>

35c, $68 \%, \alpha>96 \%$

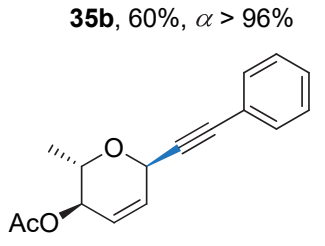

35d, $65 \%, \alpha>96 \%$

图式 14 炔基铜作为 Ferrier I 型反应的亲核试剂

Scheme 14 Copper(I) alkyne as the nucleophile for Ferrier I reaction

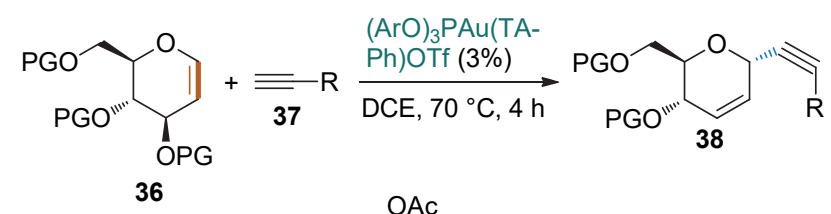<smiles>CC(=O)OCC1O[C@H](C#Cc2ccc3c(c2)CC[C@@H]2C3CCC3(C)C(=O)CC[C@H]23)C=CC1OC(C)=O</smiles>

38a, $83 \%, \alpha: \beta=98: 2$<smiles>Clc1cccc(C#C[C@@H]2C=C[C@@H](OBr)[C@@H](COc3ccccc3)O2)c1</smiles>

38c, $70 \%, \alpha: \beta=95: 5$
$38 \mathbf{b}, 64 \%, \alpha: \beta=95: 5$<smiles>CC(=O)OC[C@H]1O[C@H](C#Cc2ccccc2)C=C[C@@H]1O[SbH3]</smiles>

38d, $63 \%, \alpha: \beta=99: 1$

图式 15 炔基金作为 Ferrier I 型反应的亲核试剂 Scheme 15 Gold alkyne as the nucleophile for Ferrier I reaction

Shah 课题组 ${ }^{[19]}$ 提出一种新型的无金属催化策略 (Scheme 16). 他们以 TMSOTf 为催化剂, 原位生成炔基 三甲基硅烷，进而与糖烯发生 Ferrier I 型反应，生成高 $\alpha$ 立体选择性的炔基碳苷. 张剑波课题组 ${ }^{[20]}$ 也报道了类 似的结果.

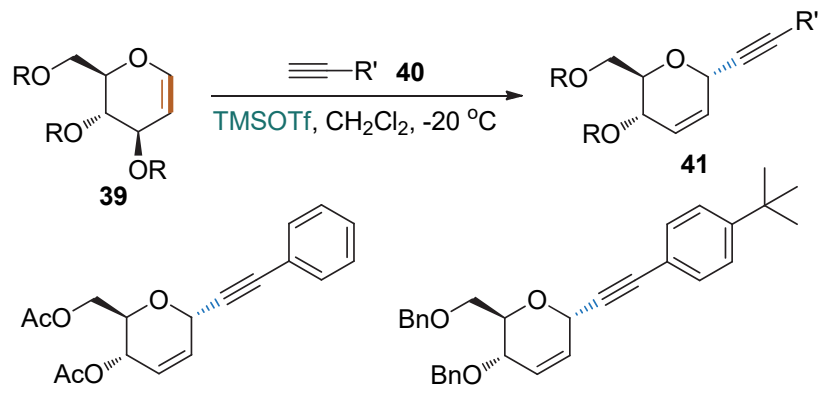

41a, $80 \%, \alpha / \beta$ 99:1<smiles>CC(=O)OC[C@H]1O[C@@H](C#Cc2ccccc2)C=C[C@@H]1OC(C)=O</smiles>

41b, $90 \%, \alpha / \beta>99: 1$

41c, $82 \%, \alpha / \beta>99: 1$<smiles>CC(=O)O[C@H]1C=C[C@@H](C#Cc2ccccc2)O[C@@H]1C</smiles>

41d, $80 \%, \alpha / \beta>99: 1$

图式 16 炔基三甲基硅烷作为 Ferrier I 型反应的亲核试剂 Scheme 16 Alkynyltrimethylsilane as the nucleophile for Ferrier I reaction

\section{4 富电子的不饱和烃作为亲核试剂的 Ferrier I 型反 应}

2014 年, 刘学伟课题组 ${ }^{[21]}$ 以三氯化铁为催化剂, 实 现了 $\beta$-酮酸的 Ferrier I 型碳苷化反应(Scheme 17); 烯醇 负离子为该反应的实际亲核试剂. 该反应条件相对温 和, 有较好的底物适用性, 但立体选择性并不是很好.
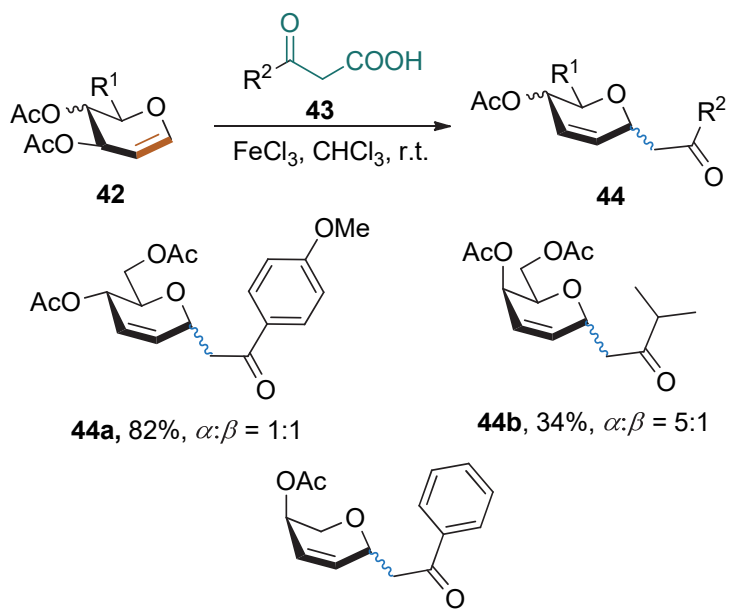

44c, $51 \%, \alpha: \beta=1: 2$

图式 $17 \beta$-酮酸作为 Ferrier I 型反应的亲核试剂 Scheme $17 \beta$-Keto acid as the nucleophile for Ferrier I reaction

2017 年, Mukherjee 课题组 ${ }^{[22]}$ 以 TBSOTf 为路易斯 酸, DIPEA 为碱，实现了芳基乙酮与糖烯的 Ferrier I 型 
碳苷化反应，以较高的产率和 $\alpha$ 立体选择性得到了苯丙 二氢吡喃碳苷(Scheme 18). 该方法还适用于克级规模 的制备.

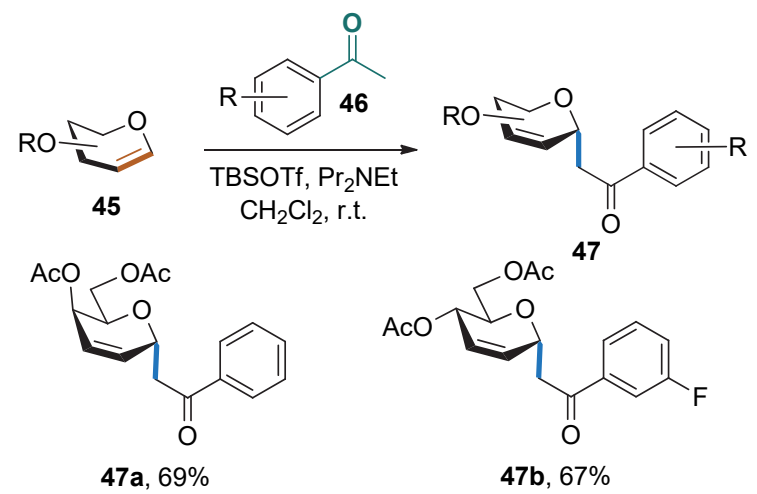

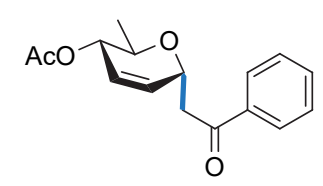

47c, $49 \%$

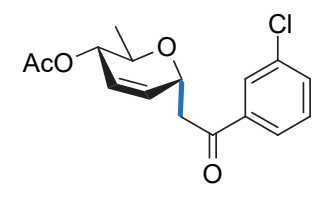

47d, $53 \%$
图式 18 芳基乙酮作为 Ferrier I 型反应的亲核试剂 Scheme 18 Aryl ethyl ketone as the nucleophile for Ferrier I reaction

Yadav 课题组 ${ }^{[23]}$ 发现, 在 $\mathrm{InBr}_{3}$ 的存在下, 苯胺上的 邻位碳可与糖烯的 1 位相连, 而氮原子则与糖烯的 3 位 相连，生成高立体选择性的 1- $C, 3-N$-双环化合物 (Scheme 19). 该反应条件温和, 具有较宽的底物适用性 和高立体选择性; 但对于生成 $\mathrm{C}-\mathrm{C}$ 和 $\mathrm{C}-\mathrm{N}$ 键的先后 顺序，作者并没有进行深入研究.

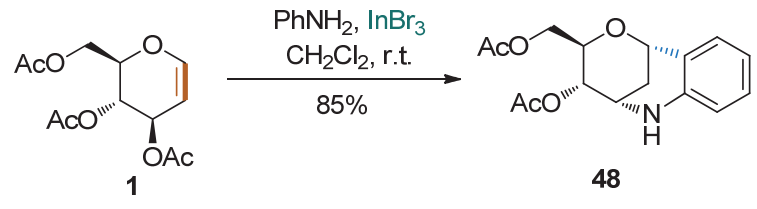

图式 19 芳胺作为反应的亲核试剂

Scheme 19 Arylamine as the nucleophile for the reaction

Sridhar 课题组 ${ }^{[24]}$ 发现在 TMSOTf 存在时, 3 位脱氧 的糖烯能两两相连生成 $\beta-1,2$ 连接的糖类化合物 (Scheme 20). 作者认为在酸的作用下糖烯可形成半椅 式构象中间体 50 , 另一分子糖烯的双键可进攻该中间 体的端位, 得到 $\beta-1,2$ 连接的假寡糖. 产物具有很好的 $\beta$ 立体选择性, 且可以进一步转化生成一系列天然碳苷片 段.

\section{5 烯丙基钯络合物作为亲电中心的 Ferrier I 型碳苷 化反应}

糖烯或其衍生物与钯生成的烯丙基钯络合物也可 发生烯丙基碳正离子类似的反应 ${ }^{[25 \mathrm{a}-25 \mathrm{~b}]}$. 2013 年, 刘学 伟课题组 ${ }^{[25 c]}$ 利用 3 -烷/芳酰基乙酸酯保护的糖烯为底
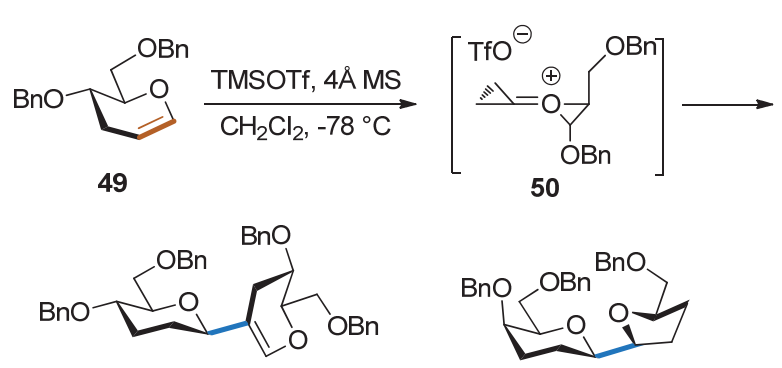

51
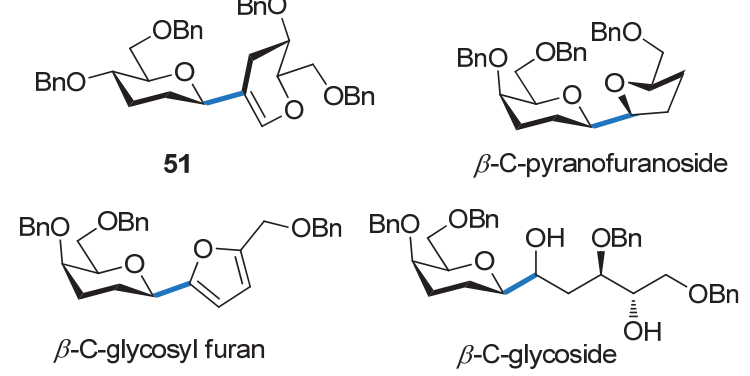

图式 20 糖烯自身作为碳苷化反应的亲核试剂

Scheme 20 Glycal as the nucleophile for $C$-glycosylation

物, 在 $\operatorname{Pd}(\mathrm{OAc})_{2} / \mathrm{DiPPF}$ 的催化下生成烯丙基钯络合物, 继而发生脱羧偶联, 得到 2,3-不饱和的烷基碳苷 (Scheme 21a). 对于 3,6-顺式的底物，该反应有非常好的 立体选择性; 而对于 3,6-反式底物, 则生成 $\alpha \beta$ 混合物. 随后, 刘学伟课题组 ${ }^{[25 \mathrm{~d}]}$ 用 $\mathrm{NHC}$ 将醛进行极性反转后也 可与烯丙基钯络合物进行反应, 得到相应的碳苷 (Scheme 21b).

(a)

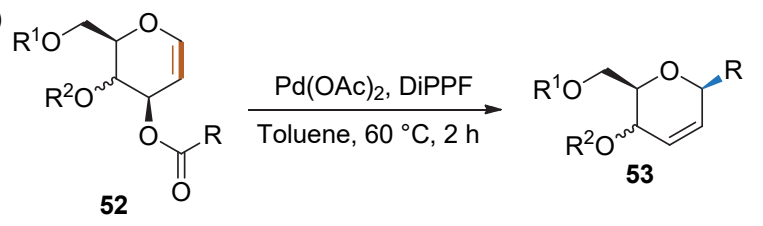<smiles>CC(=O)CC1C=CC(O)C(COCc2ccccc2)O1</smiles><smiles>CC(=O)CC1C=CC([18OH])[C@H](COC(C)(C)C)O1</smiles><smiles>COC1C=CC(CC(C)=O)OC1COC(C)(C)C</smiles><smiles>CC(C)(C)C1OCC2OC(CC(=O)c3ccccc3)C=CC2O1</smiles>

(b)<smiles>CCOC(=O)[C@@H]1C=CO[C@@H]2COC(c3ccccc3)O[C@H]21</smiles><smiles>O=Cc1ccccn1</smiles><smiles></smiles><smiles>O=C(C1=CCC2OC(c3ccccc3)OC[C@H]2O1)c1ccccn1</smiles>

图式 21 分子内的脱羧偶联反应

Scheme 21 Intramolecular decarboxylative coupling reaction

2017 年, 刘学伟课题组 ${ }^{[25 \mathrm{e}]}$ 用葡萄糖烯、磷叶立德 和醛作为底物, $\mathrm{Pd}(\mathrm{OAc})_{2} / 1,4$-二苯基膦丁烷(DPPB)为催 化剂, 实现了 2,3-不饱和的乙烯基碳苷的制备(Scheme 
22). 该反应的第一步也是形成烯丙基钯络合物，继而 发生磷叶立德的反面进攻和后续的 Wittig 反应. 可根据 加入醛基的不同得到不同构型的乙烯基碳苷. 当加入含 有吡啶基的醛时, 得到 $E$ 构型的乙烯基碳苷; 加入不含 有吡啶基的醛时，则得到 $Z$ 构型的乙烯基碳苷.
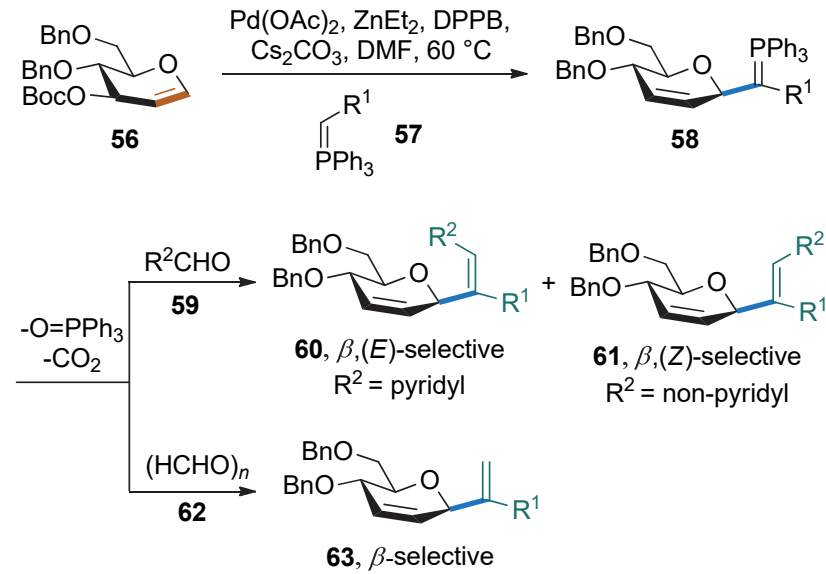

图式 22 膦叶立德作为 Ferrier I 型反应的亲核试剂

Scheme 22 Ylide as the nucleophile for Ferrier I reaction

2019 年, 张强课题组 ${ }^{[26]}$ 以 $\alpha, \beta$-不饱和内酯和 3,4-碳 酸酯保护的半乳糖烯为底物, 在 $\operatorname{Pd}(\mathrm{OAc})_{2}$ 的催化下, 实 现了 $\alpha, \beta$ 不饱和内酯的 $\gamma$-位甲基与端基碳的偶联, 生成 了 2,3-不饱和碳苷(Scheme 23). 该反应是烯丙基钯络合 物与 $\alpha, \beta$-不饱和内酯负离子的 Ferrier I 型碳苷化反应. 香豆素类化合物也可以作为该反应的底物. 该反应得到 的碳苷为 $\beta$ 构型.
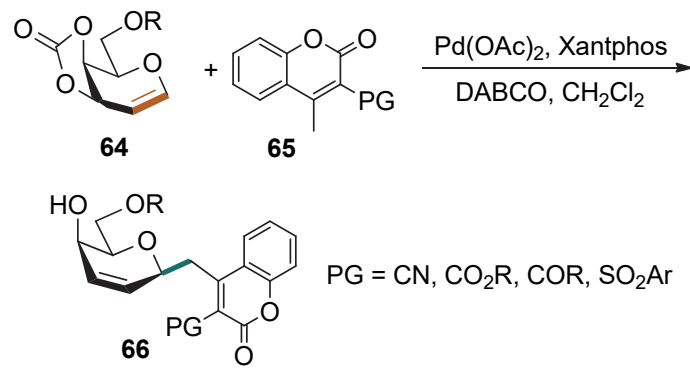

图式 $23 \alpha, \beta$ 不饱和内酯作为 Ferrier I 型反应的亲核试剂 Scheme $23 \alpha, \beta$-Unsaturated lactone as the nucleophile for Ferrier I reaction

\section{Heck 型碳苷化反应}

1968 年, $\mathrm{Heck}^{[27]}$ 报道了卤代烃与碳碳双键在钯催 化剂和碱的存在下进行偶联生成取代烯烃的反应, 被称 为 Heck 反应. Heck 反应已成为构筑 $\mathrm{C}-\mathrm{C}$ 键最有效的方 法之一. 糖烯中的双键也能与 $\mathrm{M}-\mathrm{Ar}$ 发生配位, 得到的 中间体可继续发生 $\beta$-氢消除或 $\beta$-杂原子消除得到不同 的碳苷产物，这些反应被称之为 Heck 型碳苷化反应; 由于 3 位保护基的位阻效应，一般都是生成 $\alpha$-构型的产 物. 在过去的五十年里, 化学家基于不同的偶联试剂,
发展了一系列 Heck 型碳苷化新方法(Scheme 24).

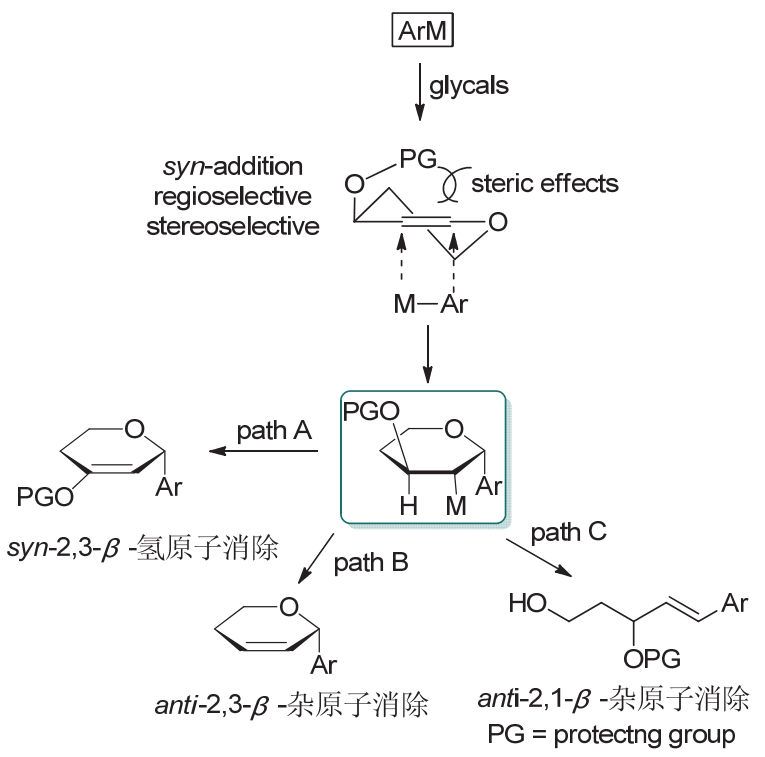

图式 24 Heck 型碳苷化反应的历程

Scheme 24 Mechanism of Heck-type $C$-glycosylation

\section{1 有机卤作为偶联试剂}

Heck 型偶联反应在糖环上的应用是从简单烯醇醚 开始的. 早在 1979 年, Daves 课题组 ${ }^{[28]}$ 就对卤代芳烃和 烯醇醚的 Heck 偶联反应进行了深入研究(Scheme 25). 他们发现碘苯和乙基乙烯基醚发生偶联反应时得到 1乙氧基乙苯和两种构型的 2-乙氧基乙苯, 而碘苯与二氢 吡喃反应得到单一的苯基取代产物.

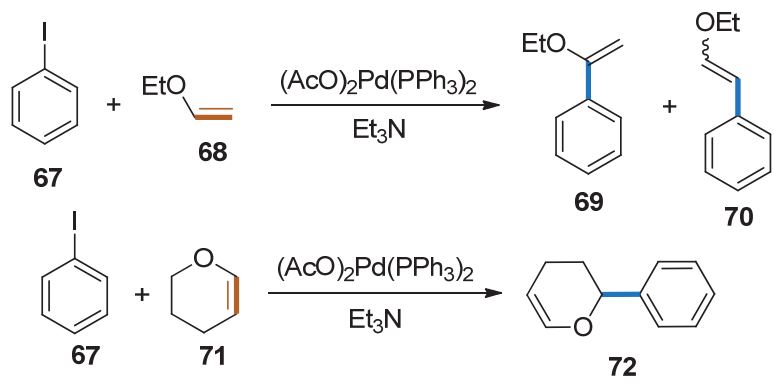

图式 25 烯醇醚参与的 Heck 偶联反应

Scheme 25 Heck coupling reaction with enol ether

Heck 型碳苷化反应最早用于一些具有特定生物活 性的分子的合成，硅基保护的呋喃型糖烯和吡喃型糖烯 均可作为底物参与反应. 1990 年, Daves 课题组 ${ }^{[29]}$ 利用 碘代芳环衍生物和叔丁基二甲基硅烷基(TBS)保护葡萄 糖烯的 Heck 型碳苷化反应成功合成了芳环类碳苷 (Scheme 26a), 该类化合物具有抗菌活性. 1992 年, Daves 课题组 ${ }^{[30]}$ 用 5-碘脲醛和呋喃核糖烯作为底物进行 Heck 偶联反应(Scheme 26b), 得到的烯醇硅醚产物经脱 硅和还原操作后得到 2-脱氧假尿苷。该化合物具有艾滋 病病毒(HIV)逆转录酶抑制活性. 
(a)

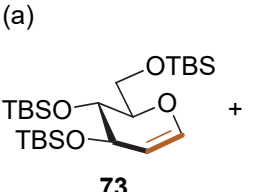<smiles>CCc1ccc2c(c1)c(=O)oc1c(I)ccc(OC)c12</smiles>

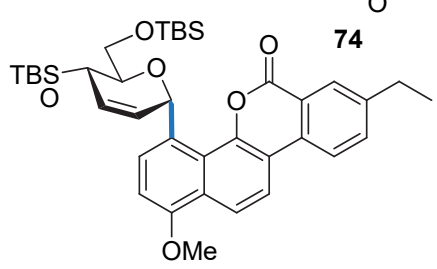

(b)

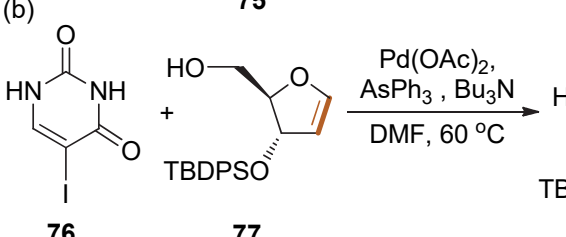

76

77

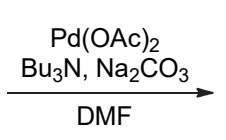<smiles>O=c1[nH]cc(C2CC(O)C(CO)O2)c(=O)[nH]1</smiles>

80<smiles>O=C1CC(c2c[nH]c(=O)[nH]c2=O)OC1CO</smiles>

78 (a) OTBS

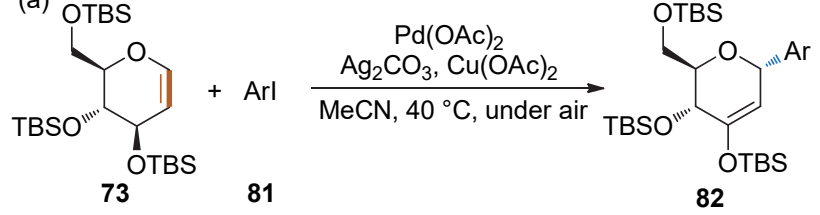

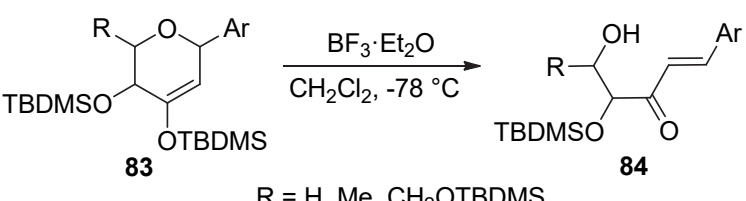

(b)

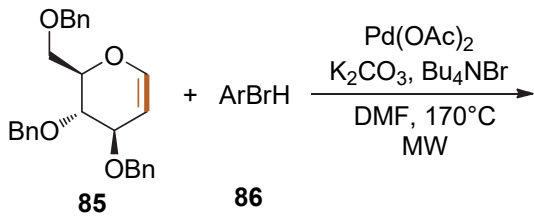<smiles>O=C(Br)C1=C[C@@H](Br)OC(COCc2ccccc2)C1[Hg]Br</smiles>

(c)<smiles>OCCC1OC=CC(Br)C1OCc1ccccc1</smiles>

$\mathrm{Pd}(\mathrm{OAc})_{2}$ $\underset{\mathrm{K}}{\stackrel{\mathrm{K}_{2} \mathrm{CO}_{3}, \mathrm{dppp}}{\mathrm{DMF}} 80^{\circ} \mathrm{C}}$

图式 26 有机卤参与的生物活性分子的合成

Scheme 26 Synthesis of bioactive compounds from organic halides

2009 年, 本课题组和杨劲松课题组分别用芳基碘 (Scheme 27a) ${ }^{[31]}$ 和芳基溴(Scheme 27b ${ }^{[32]}$ 作为底物, 在 $\mathrm{Pd}(\mathrm{OAc})_{2}$ 的催化下高效合成了 $\alpha$-芳基碳苷. 其中, 当使 用芳基溴作为底物时, 反应需要在微波照射条件下进 行. 2019 年, Savic 课题组 ${ }^{[33]}$ 利用本课题组的方法合成芳 基碳苷, 并在此基础上发展了路易斯酸促进的开环反 应, 立体选择性地合成了一些具有抗癌活性的 $\alpha, \beta$-不饱 和酮. 2013 年, 李英霞课题组 ${ }^{[34]}$ 用碘乙烯糖底物与糖烯 进行 Heck 偶联反应, 以较高的收率得到了碳苷假糖 (Scheme 27c).

\section{2 有机硼作为偶联试剂}

有机硼也可以作为 Heck 型碳苷化反应的底物. 2001 年, Maddaford 课题组 ${ }^{[35 a]}$ 以乙腈为溶剂, 在 $\mathrm{Pb}-$ $(\mathrm{OAc})_{2}$ 的催化下, 实现了全乙酰基保护的葡萄糖烯与苯 嗍酸的偶联反应, 以 $80 \%$ 的收率得到了 2,3 不饱和的 $\alpha-$ 苯基碳苷产物(Scheme 28). 他们还以 $\alpha, \beta$-不饱和酮为反 应物, 得到了酮式碳苷产物 ${ }^{[35 b]}$. 在此基础上, 2005 年 Figuera 小组 ${ }^{[36]}$ 观察到了使用芳基硼酸与全乙酰糖烯偶 联反应时某些条件下会伴有 $a n t i-2,1-\beta$-杂原子消除的开 环产物生成, 但未见可控生成开环产物的报道. 2020 年,

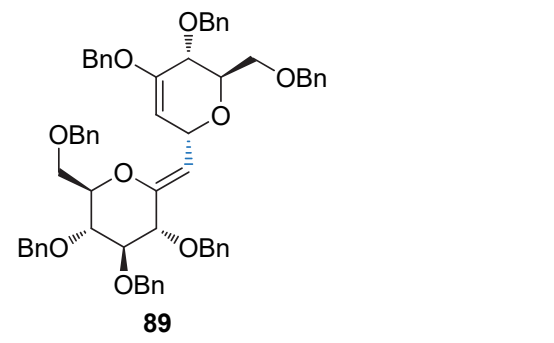

图式 27 有机卤参与的 Heck 偶联反应

Scheme 27 Heck coupling reaction with organic halides

姚辉课题组 ${ }^{[37]}$ 利用 White 催化剂实现了 3,4-碳酸酯保护 糖烯的anti-2,3- $\beta$-杂原子消除反应，得到了 2,3 不饱和的 $\alpha$-苯基碳苷.

2009 年, 本课题组 ${ }^{[38]}$ 利用芳基硼酸与全 TBS 保护 的 1,2-吡喃糖烯的氧化 Heck 偶联反应, 发展了一种 syn-2,3- $\beta$-氢原子消除反应制备芳基碳苷的方法(Scheme 29). 该反应以醋酸钯为催化剂, 在乙腈溶剂中进行, 加 入不同的氧化剂可实现催化剂的循环和产物的调控. 以 苯醌(BQ)为氧化剂时生成酮式碳苷 96; 以 $\mathrm{Cu}(\mathrm{OAc})_{2} / \mathrm{O}_{2}$ 为氧化剂时生成烯醇醚式碳苷 95; 以 2,3-二氯-5,6-二氰 基-1,4-苯醌(DDQ)为氧化剂时生成 $\alpha, \beta$-不饱和酮式碳苷 97. 该方法具有良好的立体选择性, 即苷元部分总是与 3-位取代基在糖环上的朝向相反. 2018 年, Dujardin 和 Collet 课题组 ${ }^{[39]}$ 进一步证实了该立体选择性规律. 2019 年, Arisawa 和 Nimura 课题组 ${ }^{[40]}$ 利用本课题组的方法完 成了 Spliceostatin A 碳苷类似物的合成. 


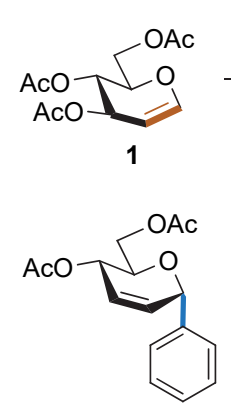

$91 \mathrm{a}, 82 \%$

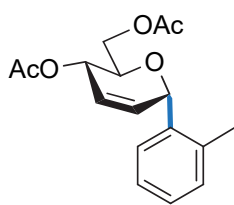

91b, $77 \%$
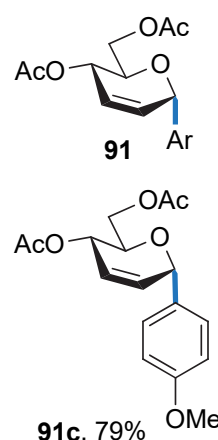

91c, $79 \%$ OMe

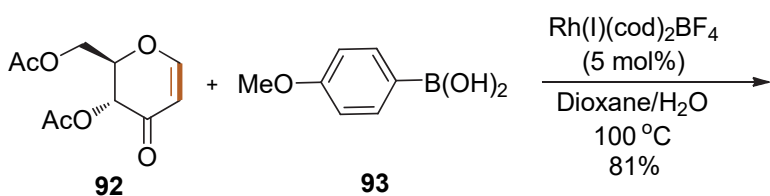<smiles>COc1ccc([C@H]2CC(=O)[C@H](OC(C)=O)[C@@H](COC(C)=O)O2)cc1</smiles>

图式 28 嗍酸参与的 Heck 偶联反应和 1,4-加成反应 Scheme 28 Heck coupling reaction and 1,4-addition reaction with arylboronic acid

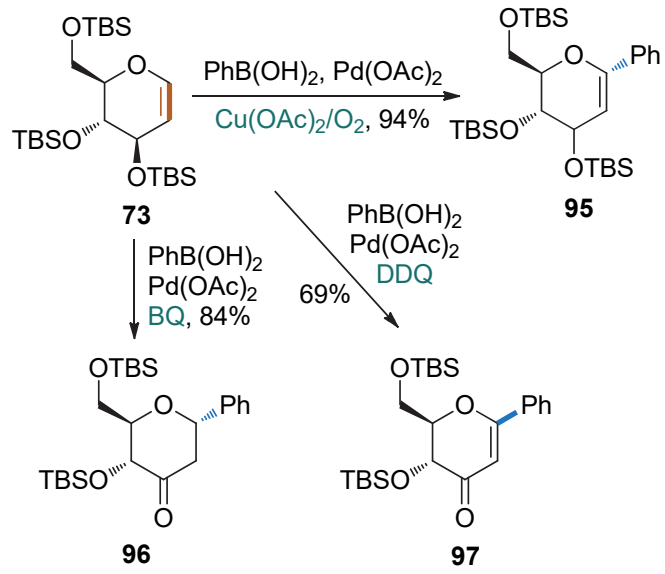

图式 29 苯嗍酸参与的 Heck 类型偶联反应

Scheme 29 Heck-type coupling reaction with arylboronic acid

2014 年, 本课题组 ${ }^{[41]}$ 发现了可控的 anti-2,1- $\beta$-杂原 子消除反应，并利用开环一关环策略通过控制反应条件 实现了2-脱氧碳苷的立体选择性合成(Scheme 30). 糖烯 和芳基嗍酸在微波加热 $170{ }^{\circ} \mathrm{C}$ 条件下，通过镍催化的 Heck 型偶联/anti-2,1- $\beta$-杂原子消除反应得到开环产物; 该中间体在不同的条件下可以选择性关环得到不同的 碳苷. 当以 $\mathrm{Sc}(\mathrm{OTf})_{3}$ 作为催化剂时得到以 $\beta$ 构型为主的 碳苷; 而以 $\mathrm{Ph}_{3} \mathrm{P} \cdot \mathrm{HBr}$ 为催化剂时则得到 $\alpha$ 构型为主的 产物. 通过 NBS 的关环脱溴可得到 $\alpha$ 构型的 2-脱氧碳 苷; 而利用 $\mathrm{PhSeCl}$ 的关环脱硒则得到 $\beta$ 构型的 2-脱氧 碳苷.

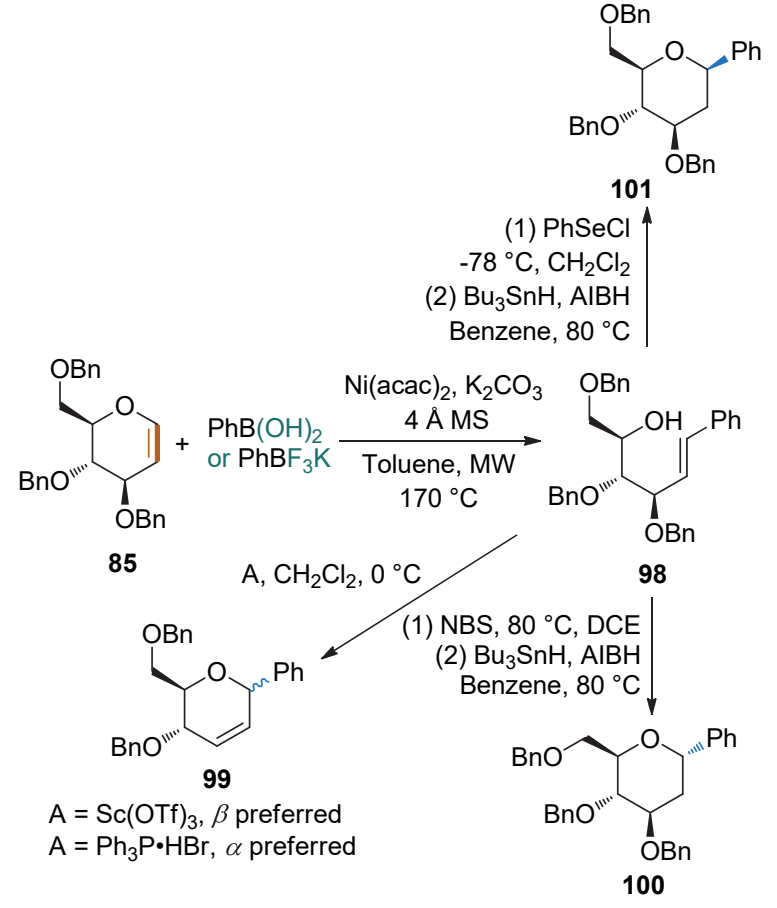

图式 30 开环一关环策略合成碳苷

Scheme 30 "Ring opening-ring closure" strategy for the synthesis of $C$-glycoside

2015 年, Mukherjee 课题组 ${ }^{[42]}$ 发现在 2,2,6,6-四甲基 哌啶氮氧化物(TEMPO)存在下，1 equiv.糖烯和 2 equiv. 硼酸在醋酸钯的催化下生成饱和的 1,2-双芳基碳苷。该 方法对给电子硼酸和吸电子芳基硼酸都能耐受，也能适 用于其它糖型(Scheme 31).

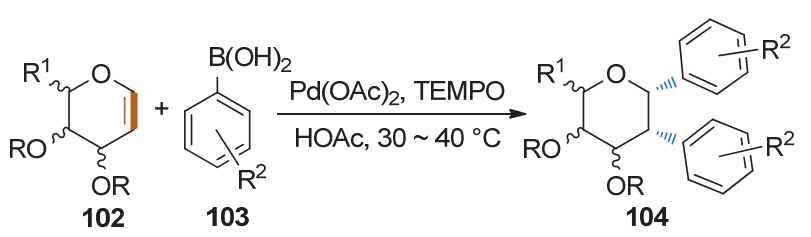<smiles>CC(=O)OC[C@H]1O[C@@H](c2ccccc2)[C@H](c2ccccc2)[C@H](OC(C)=O)[C@@H]1OC(C)=O</smiles>

$104 a, 80 \%$<smiles>CC(C)(C)OC[C@H]1O[C@H](c2ccccc2)[C@H](c2ccccc2)[C@H](OC(=O)OC(C)(C)C)[C@H]1OC(=O)OCc1ccccc1</smiles>

104c, $70 \%$<smiles>CC(=O)OC[C@H]1O[C@@H](c2ccc3c(c2)OCO3)[C@H](c2ccc3c(c2)OCO3)[C@H](OC(C)=O)[C@@H]1O[Na]</smiles><smiles>CC(=O)O[C@H]1[C@H](c2ccccc2)[C@@H](c2ccccc2)O[C@H](C)[C@H]1OC(C)=O</smiles>

图式 31 TEMPO 作为 Heck 偶联反应的氧化剂 Scheme 31 Heck coupling reaction with TEMPO as the oxidant

\section{3 芳胺/肼作为偶联试剂}

芳肼也可以作为偶联试剂. 2013 年, 刘学伟课题 
组 ${ }^{[43]}$ 发展了芳基肼与葡萄糖烯的偶联反应(Scheme 32). 在 $\mathrm{Pd}(\mathrm{OAc})_{2}$ 的催化和氧气的存在下, 该反应可高收率 地实现 2,3-不饱和 $\alpha$-芳基碳苷的合成. 他们还发现, 糖 烯 3 位的取向不是 1,3 反式立体选择性的唯一决定因素. 因为, 将葡萄糖烯的 3 位异构化之后反应仍可顺利进行, 但得到的是 $\alpha, \beta$ 异构体的混合物, 且随着芳基取代基的 不同立体异构比例稍有差异.
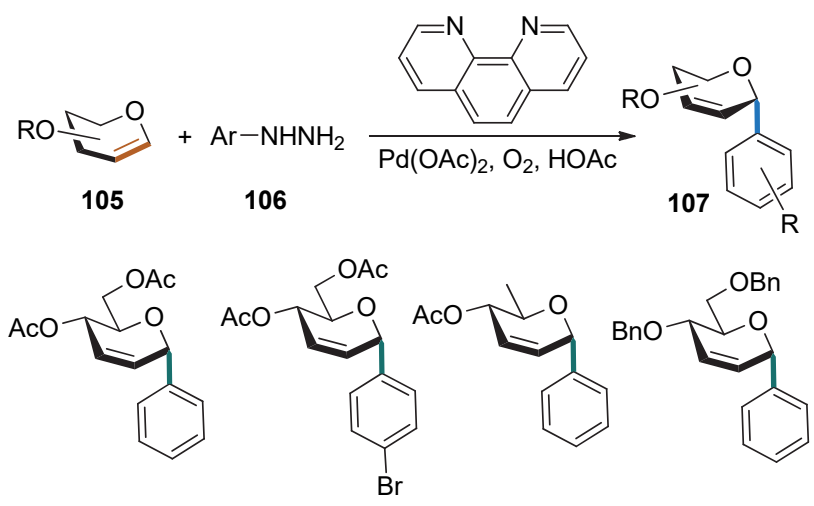

$107 a, 90 \%$

$107 b, 85 \%$

$107 \mathrm{c}, 83 \%$

$107 d, 72 \%$

图式 32 芳肼参与的 Heck 偶联反应

Scheme 32 Heck coupling reaction with aromatic hydrazine

2018 年, 本课题组 ${ }^{[44]}$ 发展了一种以芳胺为偶联剂、 立体可控的 2-脱氧芳基碳苷的合成方法. 用芳胺作为原 料, 在 $\mathrm{NOBF}_{4}$ 的作用下原位生成芳基重氮盐, 继而在 $\operatorname{Pd}(\mathrm{dba})_{2}$ 的催化下与糖烯发生偶联反应, 生成 $\alpha$ 构型的 3-羰基碳苷. 如果在反应结束后加入四氟硼酸二乙醚溶 液则得到 $\beta$ 构型的产物. 该方法从芳胺出发原位制备重 氮盐, 比直接用重氮盐更加安全(Scheme 33). 此外, 该 方法仅通过简单条件的改变, 实现了立体选择性的调 控, 操作简单、实用性高. 该方法还可用于含有 3-羰基 碳苷骨架的天然产物的合成.

随后, Kandasamy 课题组 ${ }^{[45]}$ 也报道了在钯的催化下 重氮盐和糖烯偶联生成 $\alpha$ 构型的酮式碳苷的反应. 2019 年, 该课题组还从苯胺出发, 经亚硝酸叔丁酯 $/ \mathrm{HBF}_{4}$ 原 位制得重氮盐, 然后根据 3-位取代基取向的不同调控立 体选择性.

\section{4 羧酸作为偶联试剂}

2011 年, 刘学伟课题组将偶联试剂的范围扩大到 了有机羧酸 ${ }^{[46]}$. 他们以 $\mathrm{Pd}(\mathrm{OAc})_{2} / \mathrm{PPh}_{3}$ 作为催化剂, $\mathrm{Ag}_{2} \mathrm{CO}_{3}$ 为氧化剂, 在二甲亚砜(DMSO)和 DMF 的混合 溶液中实现了芳甲酸和葡萄糖烯的脱羒偶联反应, 得到 了 $s y n-2,3-\beta$-氢消除的 2-脱氧- $\alpha$-芳基糖苷(Scheme 34). 该反应需要在加热条件下进行, 具有良好的选择性, 收 率中等.

\section{5 苯磺酰氯作为偶联试剂}

2015 年, Mukherjee 课题组 ${ }^{[47]}$ 将偶联试剂的范围扩

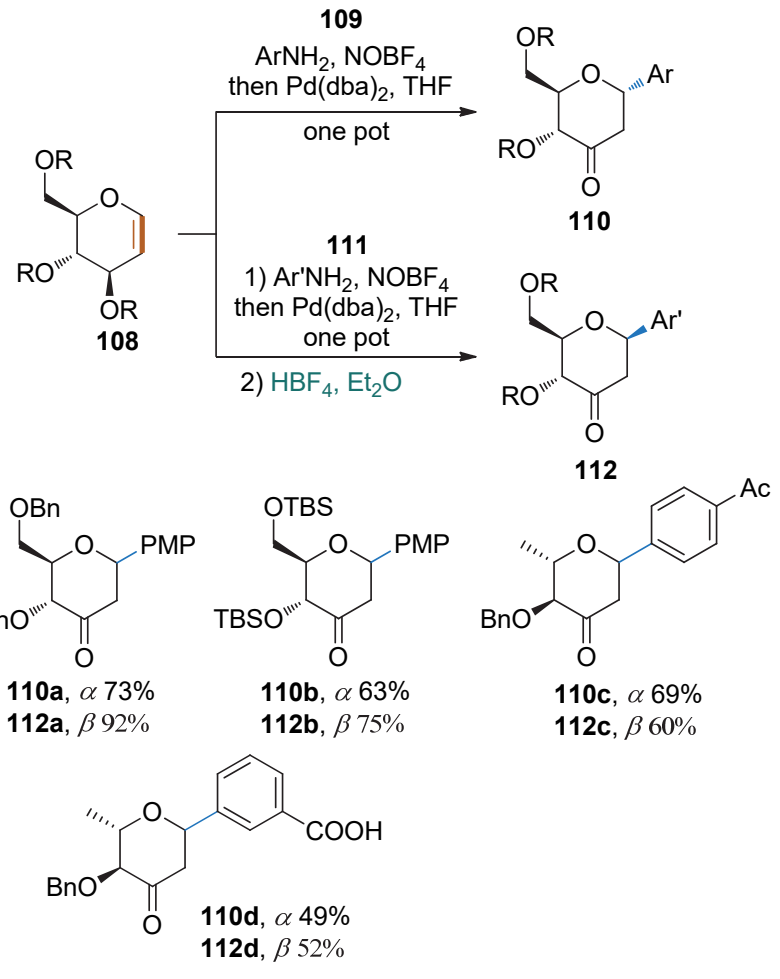

图式 33 芳胺参与的碳苷合成反应

Scheme 33 -Glycoside synthesis with aromatic amine

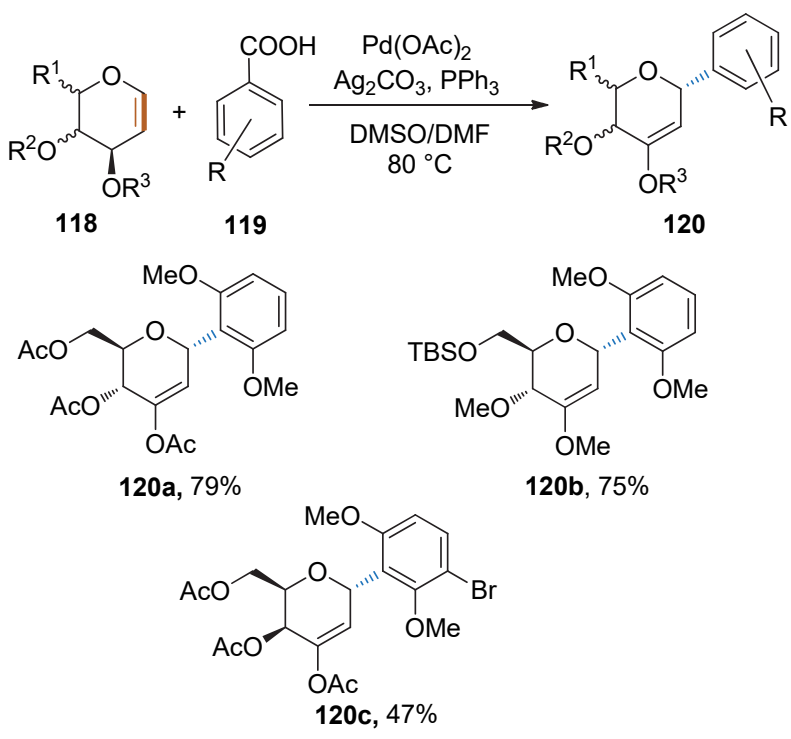

图式 34 苯甲酸参与的 Heck 偶联反应

Scheme 34 Heck coupling reaction with benzoic acid

大至苯磺酰氯. 糖烯和芳基磺酰氯在 $\mathrm{Pd}\left(\mathrm{Ph}_{3} \mathrm{P}\right)_{2} \mathrm{Cl}_{2}$ 的催 化下, 经由 anti-2,3- $\beta$-杂原子消除反应, 生成 2,3-不饱和 的芳基碳苷. 产物均为 $\alpha$ 构型, 反应收率中等(Scheme 35).

\section{6 杂环化合物作为偶联试剂}

前述 Heck 类型碳苷化反应均通过改变偶联试剂中 的离去基团来实现. 2019 年, Nishimura 课题组 ${ }^{[48]}$ 在铱/ binap 的催化下, 利用吡啶基团导向的碳氢键活化, 实 


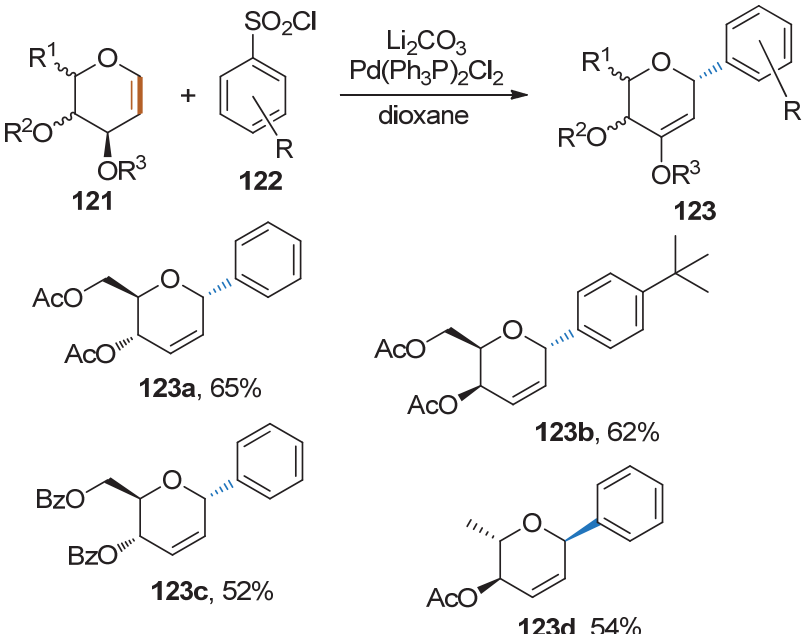

图式 35 苯磺酰氯参与的 Heck 偶联反应

Scheme 35 Heck coupling reaction with benzenesulfonyl chloride

现了 2-苯基吡啶和糖烯的 Heck 类型碳苷化反应, 得到 了饱和的 2-脱氧碳苷(Scheme 36). 更有趣的是, 不同的 手性配体可实现立体选择性的调控. (S)-binap 配体通常 得到 $\beta$-(为主)的碳苷.

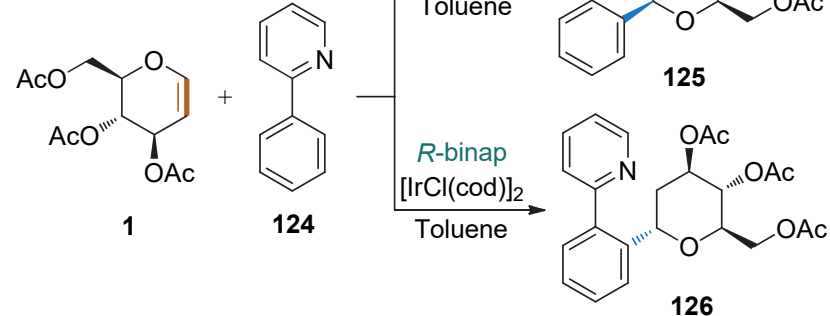

图式 36 2-苯基吡啶参与的 Heck 偶联反应 Scheme 36 Heck coupling reaction with 2-phenylpyridine

\section{3 基于 1-取代糖烯的过渡金属催化碳苷化反应}

除了 Heck 型碳苷化反应之外, 还可以在糖烯的 1 位引入活化、定位或极性反转基团, 使其更容易发生过 渡金属催化的偶联反应, 生成 1,2-不饱和的碳苷. 近二 十年来, 化学家们发展了多种基于 1-取代糖烯的碳苷化 反应.

\section{1 烯基硼作为糖基供体}

有机嗍化合物是 Suzuki 反应的理想底物. Miyaura 和 Ishiyama 课题组 ${ }^{[49]}$ 发展了 1-喼酸频那醇酯糖烯的制 备方法，并发现其可与芳基碘代物发生 Suzuki-Miyaura 偶联反应, 得到 1,2-不饱和碳苷(Scheme 37a). Parkan 和 Kotora $^{[50]}$ 课题组进一步完善了 1-硼酸频那醇酯糖烯与 芳基/烯基碘或溴的偶联反应，并将该反应拓展至 1-砋
酸糖烯(Scheme 37b). 随后, Parkan 课题组 ${ }^{[51]}$ 用 6-碘代糖 代替溴代芳基作为反应底物进行反应，实现了 1-硼酸频 那醇酯糖烯与 $\mathrm{sp}^{3}$ 碳的偶联，以较高的收率得到 $1 \rightarrow 6$ 连 接的伪二糖(Scheme 37c).

(a)

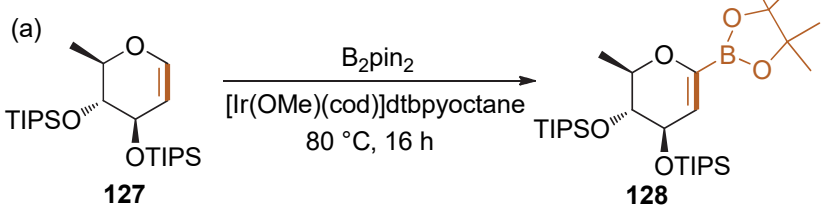

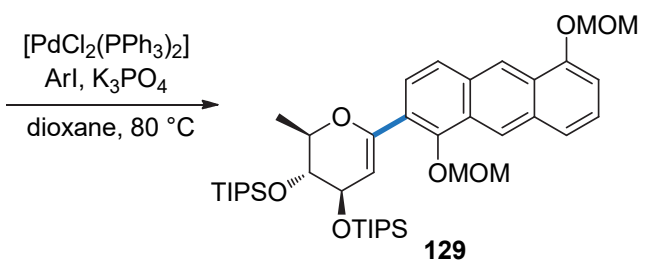

(b)

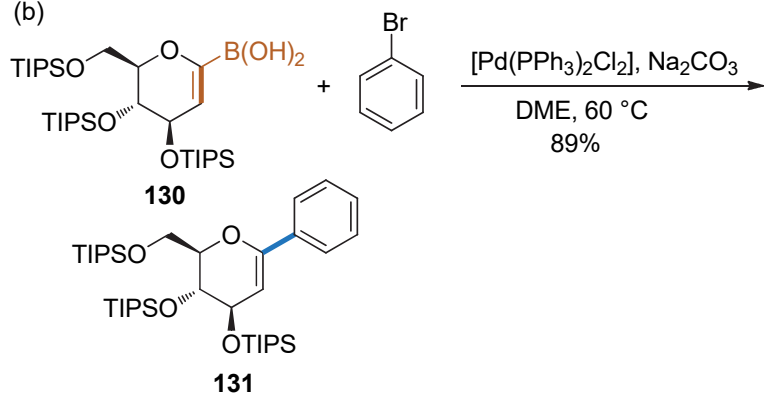

(c)
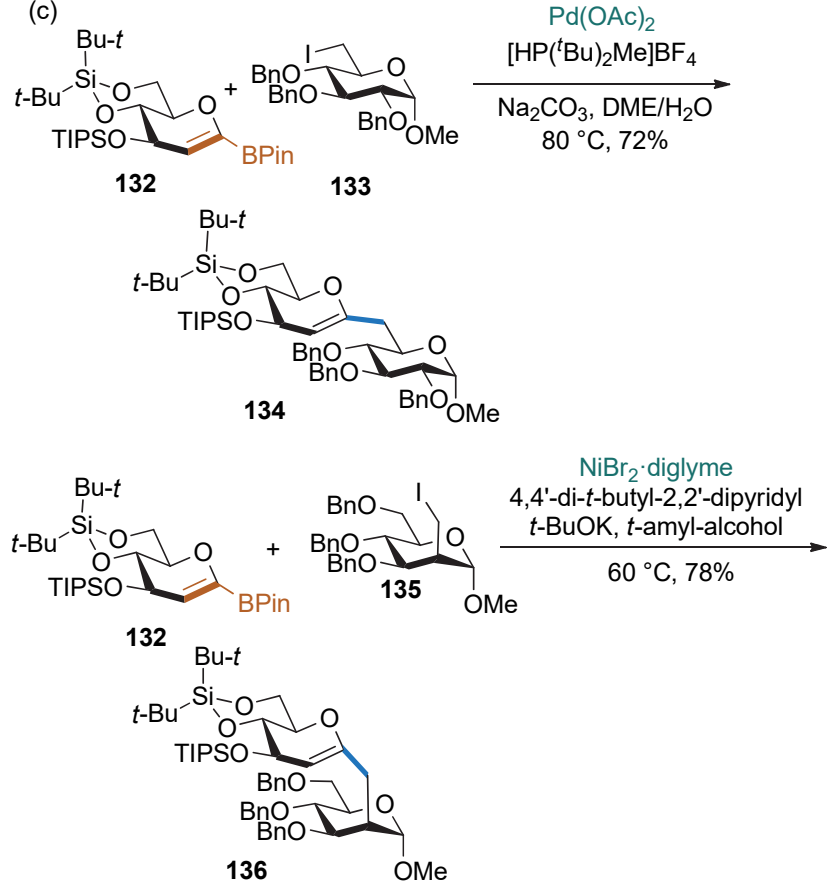

图式 37 1-嗍代糖烯作为糖基供体

Scheme 37 1-Boron-substituted glycals as the glycosyl donors

\section{2 烯基砜作为糖基供体}

烯基砜也是理想的 $\mathrm{C}-\mathrm{C}$ 键构筑前体. 2019 年，钮 大文课题组 ${ }^{[52]}$ 以 1-砜代糖烯为糖基供体, 在镍催化剂的 
作用下与芳基硼酸发生 Suzuki-Miyaura 偶联反应, 得到 了 1,2-不饱和的碳苷(Scheme 38). 该反应有良好的底物 适用性, 可用于伊格列净等药物或药物活性片段的合 成.

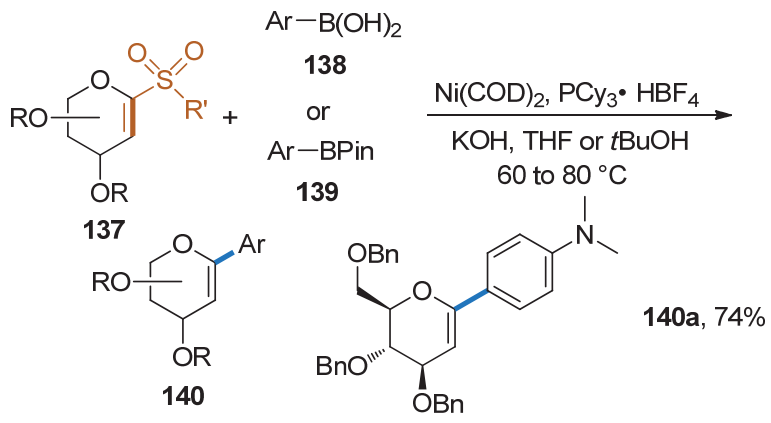

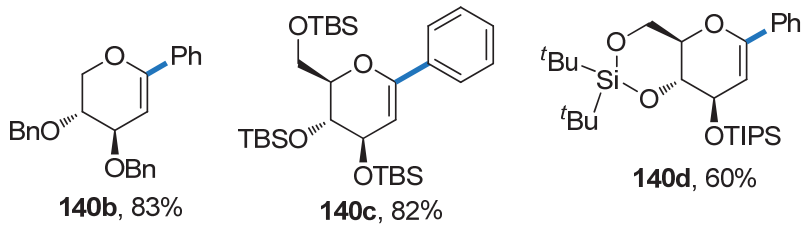

图式 38 1-砜代糖烯作为糖基供体

Scheme 38 1-Sulfone-substituted glycals as the glycosyl donors

\section{3 烯基锡作为糖基供体}

糖烯的 1-位可以被锡取代，进而通过 Stille 偶联反 应制备碳苷. 2004 年, Vogel 课题组 ${ }^{[53]}$ 发展了一种 1-锡基 取代的糖烯与芳基磺酰氯之间的 Stille 偶联反应 (Scheme 39a), 得到了 1,2-不饱和的碳苷. 2005 年,
McDonald 课题组 ${ }^{[54]}$ 以 1-锡取代的糖烯和 $\alpha$-碘代苯乙烯 作为底物, 在 $\mathrm{Pd}\left(\mathrm{PPh}_{3}\right)_{4} / \mathrm{CuI} / \mathrm{CsF}$ 的条件下进行 Stille 偶 联反应, 得到嵌合 1,3-丁二烯的碳苷产物. Danishefsky 课题组 ${ }^{[55]}$ 用大位阻的芳基溴与端基被锡取代的糖烯进 行 Stille 偶联, 得到芳基碳苷产物(Scheme 39b). 2013 年, Werz 课题组 ${ }^{[56]}$ 以 1-锡基取代的糖烯和含烯基溴取代的 葡萄糖为底物, 在 $\mathrm{Pd}\left(\mathrm{PPh}_{3}\right)_{4} / \mathrm{CuI}$ 条件下进行 Stille 偶联 反应, 以较高的收率得到 $1 \rightarrow 2,1 \rightarrow 3,1 \rightarrow 4$ 和 $1 \rightarrow 6$ 连接 的伪二糖(Scheme 39c), 为碳连接的寡糖合成提供了通 用的方法.

\section{4 烯基碘作为糖基供体}

碘作为一种很好的离去基团也可以活化糖烯. 2004 年, Tan 课题组 ${ }^{[57]}$ 利用烯烃和 9-BBN 原位生成的硼烷与 1-碘糖烯的 Suzuki 偶联反应, 在 $\mathrm{Pd}(\mathrm{dppf}) \mathrm{Cl}_{2}$ 的催化下, 实现了 1,2-不饱和烷基碳苷的合成(Scheme 40). 该反应 还可以结合羰基插入反应，得到酰基碳苷.

2016 年, 本课题组 ${ }^{[58]}$ 以端基碘取代的糖烯和 $N$-喹 啉苯甲酰胺类化合物为底物, $\mathrm{Pd}(\mathrm{OAc})_{2}$ 为催化剂和氨基 酸为配体, 经由 8-氨基喹啉(AQ)导向的碳氢键活化和 偶联，以较高的收率得到了 1,2-不饱和的芳基碳苷，并 通过简单的化学转化实现了螺环碳苷骨架和 $\beta$-碳苷的 合成. 2017 年, 本课题组 ${ }^{[59]}$ 以 $\mathrm{CuI}$ 和 $\mathrm{Pd}(\mathrm{OAc})_{2}$ 为催化剂, 通过无导向基团的碳氢键活化方式，在糖烯的 1-位引入 了杂环芳烃, 为杂环芳烃碳苷的合成提供了新的途径 (Scheme 41).

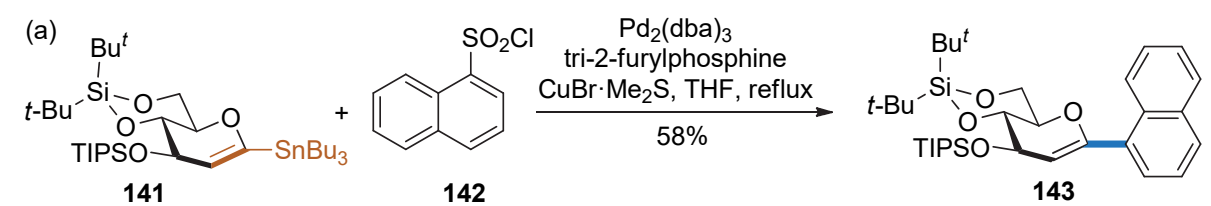

(b)
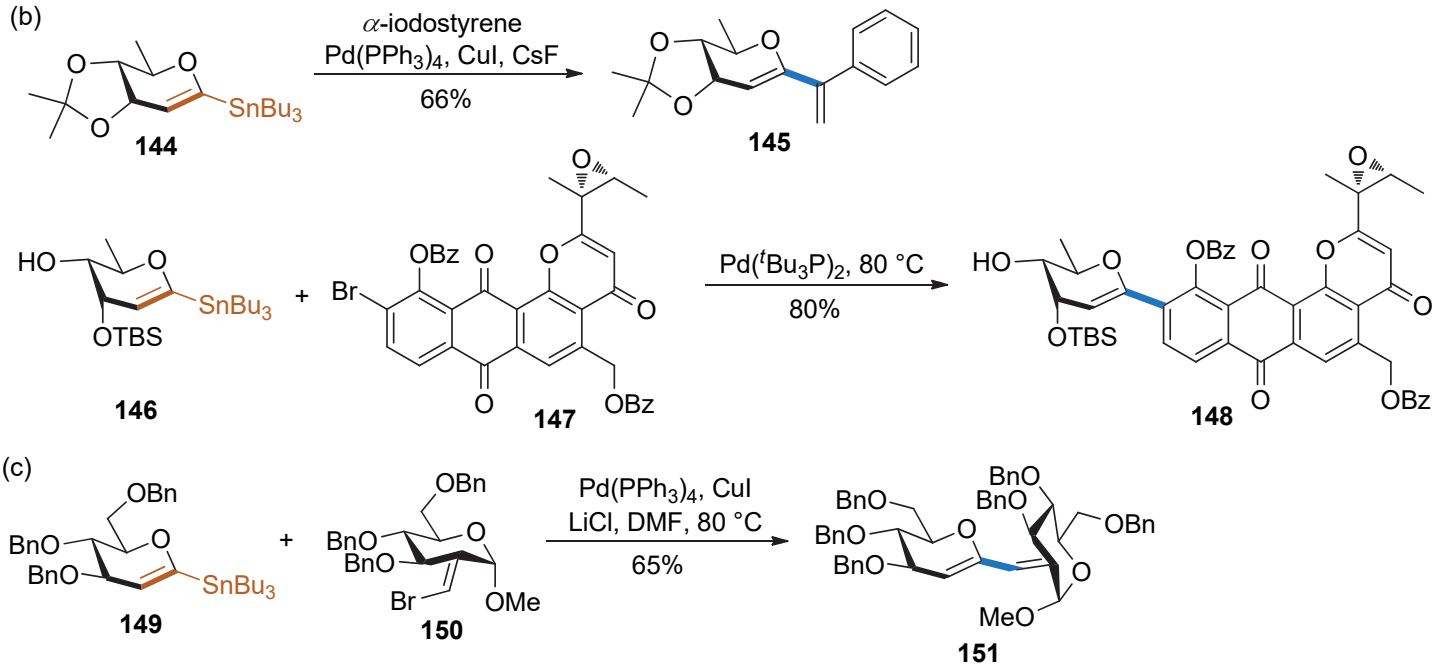

图式 391 -锡代糖烯作为糖基供体

Scheme 39 1-Stannyl-glycals as the glycosyl donors 


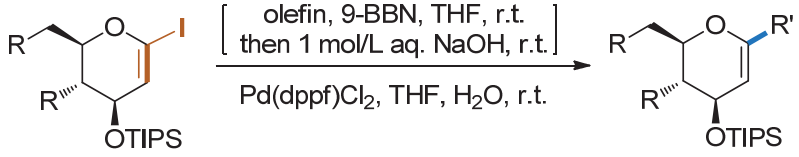

152<smiles>CCCOc1ccccc1</smiles>

153a, $81 \%$

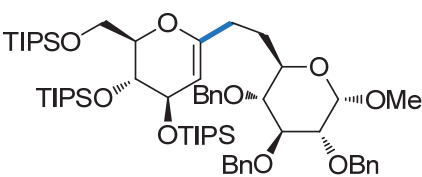

153c, $97 \%$

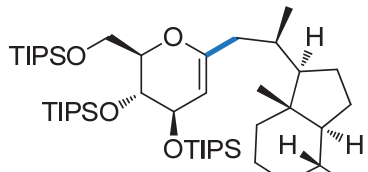

153

图式 401 - 碘糖烯和硼化合物的碳苷化反应

Scheme 40 -Glycosylation of 1-iodoglycals with boron compound
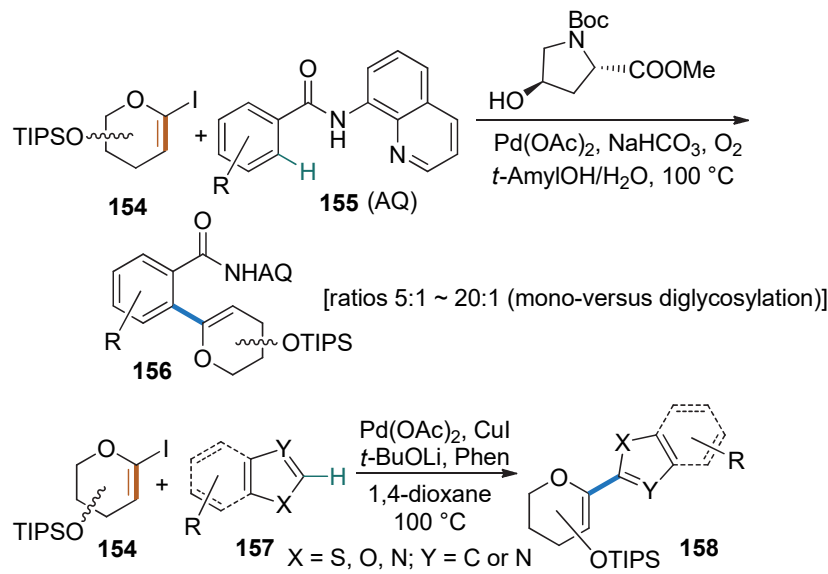<smiles>Cc1cccc(C2=CC(O)C(OC(C)(F)F)C(COC(=O)O)O2)c1C(=O)NC(C)C</smiles>

$156 a, 91 \%$

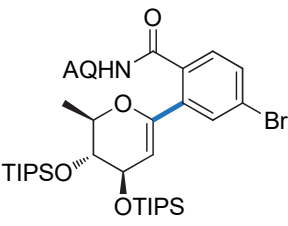

$156 \mathrm{c}, 80 \%, 10.2: 1$

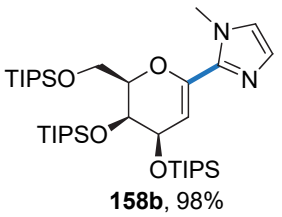

图式 41 1-碘糖烯和芳烃的碳苷化反应

Scheme $41 C$-Glycosylation of 1-iodoglycals with aromatic hydrocarbon
2019 年, 柳红和李英霞课题组 ${ }^{[60]}$ 以 8 -氨基喹啉 (AQ)作为导向基团, 实现了氨基酸 $\beta$-位 $\mathrm{sp}^{3}$ 碳的碳氢键 活化, 进而与 1-碘糖烯进行偶联反应, 得到 1,2-不饱和 的糖氨基酸(Scheme 42a). 2020 年, Ackermann 课题组 ${ }^{[61]}$ 以三唑基二甲基甲基(triazolyldimethylmethyl，TAM)作 为导向基团, 完成了类似的反应(Scheme 42b). 这些方 法有可能用于多肽的后修饰.

\section{5 烯基锌/硅作为偶联试剂}

在糖烯合成碳苷的反应中，1-锌代的糖烯也可以作 为偶联试剂参与反应. 2003 年, Auge 课题组 ${ }^{[2]}$ 利用 1-锌 代的糖烯和卤代芳烃进行 Negishi 偶联反应得到碳苷产 物(Scheme 43a). 1-硅代的糖烯也可作为偶联试剂参与 Hiyama 反应. 2012 年, Pieters 课题组 ${ }^{[63]}$ 用 1-硅代的糖烯 和卤代芳烃作为底物, 在 $\operatorname{Pd}(\mathrm{dba})_{3}$ 催化下发生偶联得到 1,2-不饱和的芳基碳苷产物(Scheme 43b).

\section{6 烯基锂作为偶联试剂}

前述糖烯都是作为亲电试剂参与反应，而端基用金 属取代的糖烯也可以作为亲核试剂参与反应. 1994 年, Parker 课题组 ${ }^{[64]}$ 利用 1-锂代的糖烯, 通过糖与酮羰基的 亲核加成反应，实现了䒬醌的双糖基化(Scheme 44a). 2008 年, Suzuki 课题组 ${ }^{[65]}$ 以 1 -锂代的葡萄糖烯和异恶唑 酮作为底物, 完成了决明子碳苷的合成(Scheme 44b).

\section{Michael 加成型碳苷化反应}

当糖烯的 2-位有硝基存在时，糖烯的双键和硝基构 成了 $\alpha, \beta$-不饱和体系，该体系可以在碱性条件下和碳负 离子进行 Michael 加成反应, 生成相应的碳苷. 1996 年 Holzapfel 课题组 ${ }^{[6]}$ 利用烯丙负离子与 2-硝基糖烯的 Michael 加成反应, 并结合烯丙基钯化学, 发展了含 1,2并环结构的碳苷合成方法. 该方法的立体选择性不是很 好, 得到 $\beta$-构型为主的产物 (Scheme 45a). 2002 年, Schmidt 和 Vankar 课题组 ${ }^{[67]}$ 以叔丁醇钾为碱, 实现了 2硝基半乳糖烯和丙二酸二甲酯等含活泼亚甲基底物的 Michael 加成反应, 以较高收率得到 $\beta$ 构型为主的碳苷 (Scheme 45b). 2005 年, Vankar 课题组 ${ }^{[68]}$ 实现了乙烯基澳 化镁等格式试剂底物的 Michael 加成反应, 并用该方法 完成了一些具有糖苷酶抑制活性的杂糖的合成(Scheme $45 \mathrm{c})^{[69]}$.

2010 年, 俞初一课题组 ${ }^{[70]}$ 将底物扩展到了烯氨酯, 完成了一系列烯氨酯类化合物与 2-硝基葡萄糖烯在碱 性条件下的 Michael 加成反应(Scheme 46a). 2012 年, 刘 学伟课题组 ${ }^{[71]}$ 利用 NHC 催化剂对醛的极性反转效应, 实现了醛与 2-硝基糖烯的偶联反应. 该方法有良好的 $\beta$ 立体选择性(Scheme 46b). 2014 年, Pannecoucke 课题 
(a)

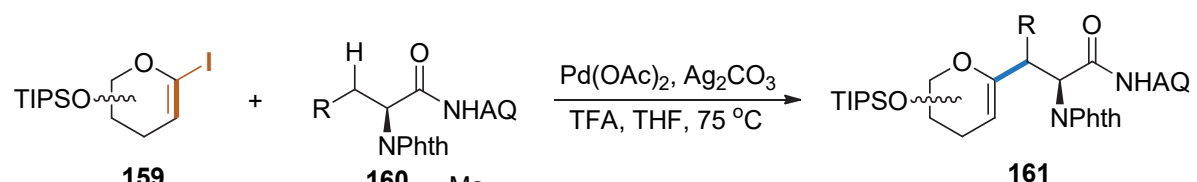

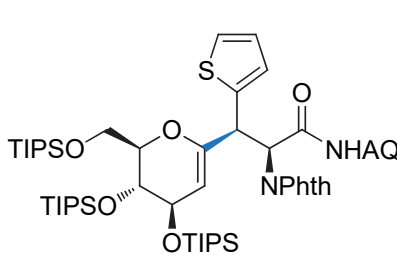

161a, $78 \%, d r>20: 1$

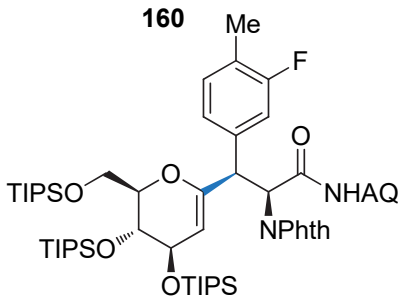

161b, $78 \%, d r=12: 1$

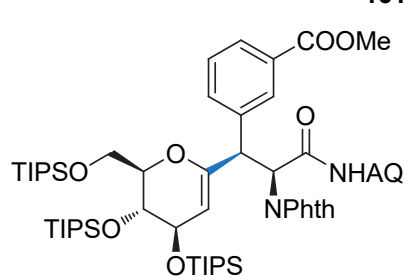

161c, $69 \%, d r=8: 1$

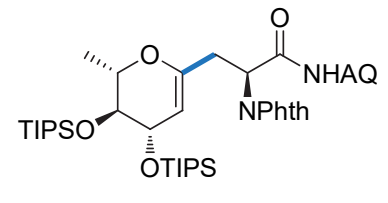

161d, $81 \%$

(b)

$$
\text { 作 }
$$<smiles>[R][Y10]NC(=O)c1cc[R1]([Y10])cc1C1=CCC([R20])CC1</smiles>

162

165<smiles>[Y10]NC(=O)[C@@H](Nc1ccccc1)C([R])C1=CCC([R20])CO1</smiles>

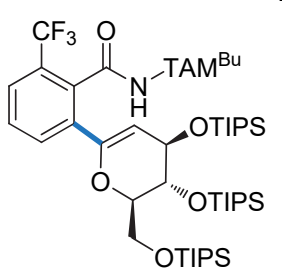

164a, $94 \%$

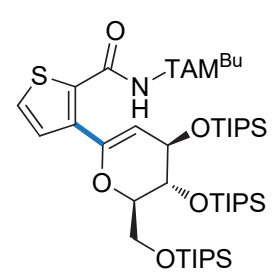

164b, $87 \%$

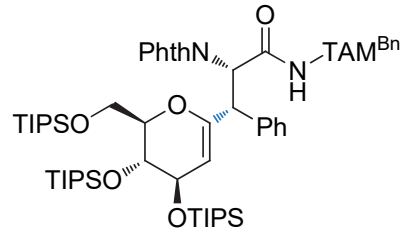

166a, $95 \%$, d.r. $>20: 1$

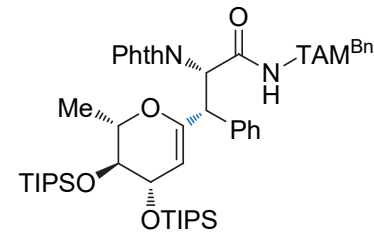

166b, $86 \%, d . r .>20: 1$

图式 42 1-碘糖烯和氨基酸的碳苷化反应

Scheme $42 C$-Glycosylation of 1-iodoglycals with amino acids

(a)

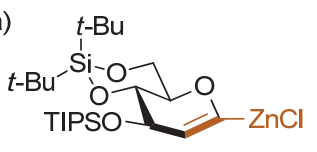

167<smiles>CCOC(=O)[C@H](Cc1ccc(I)cc1)NC(C)=O</smiles>

168

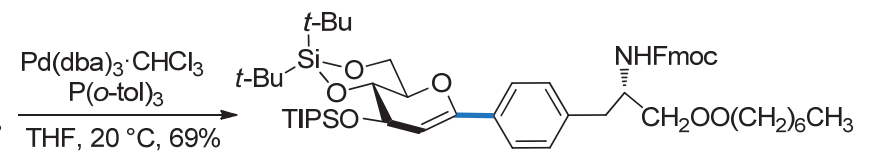

169

(b)

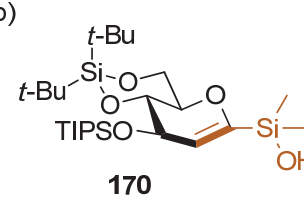<smiles>CCCOCc1cc(OCc2ccccc2)cc(OCc2ccccc2)c1I</smiles>
171

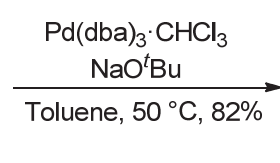

图式 43 烯基硅/锌作为偶联试剂

Scheme 43 Alkenyl silicon/zinc as the coupling substrate

组 ${ }^{[72]}$ 实现了有机锂与 2-硝基糖烯的 Michael 加成反应, 得到 $\beta$ 型的碳苷产物(Scheme 46c). 随后, Pannecoucke 课题组 ${ }^{[73]}$ 和 Ruijter 课题组 ${ }^{[74]}$ 分别实现了含氟的硅试剂 和锂试剂与 2-硝基糖烯的 Michael 加成反应, 得到了氟 代碳苷(Scheme 46d).

\section{5 自由基加成型碳苷化反应}

理论上, 碳自由基也可以对糖烯进行加成 ${ }^{[75]}$. 但碳 自由基通常会加成到糖烯的 2-位. 当糖烯的 2-位有取代
基的时候，碳自由基可加成到端位，生成碳苷. Leclerc 课题组 ${ }^{[76]}$ 以 $\mathrm{BEt}_{3}$ 作为自由基引发剂, 使溴代物裂解得 到 $\mathrm{CF}_{2} \mathrm{CO}_{2} \mathrm{Et}$ 和 $\mathrm{CF}_{2} \mathrm{Br}$ 自由基，从而与 2-苠氧基糖烯发 生自由基加成反应, 生成相应的氟代碳苷. 当用葡萄糖 烯作为底物时, 得到 $\alpha: \beta=3: 1$ 的混合物; 而用半乳糖 烯作为底物时可得到 $\alpha$ 构型的产物(Scheme 47a). Baran 课题组 ${ }^{[77]}$ 利用铁催化剂, 在苯基硅烷的存在下, 通过氢 自由基对葡萄糖烯 2-位的加成使其形成糖自由基，继而 对丙烯酸甲酯进行自由基加成, 得到 $\alpha-2$-脱氧碳苷 


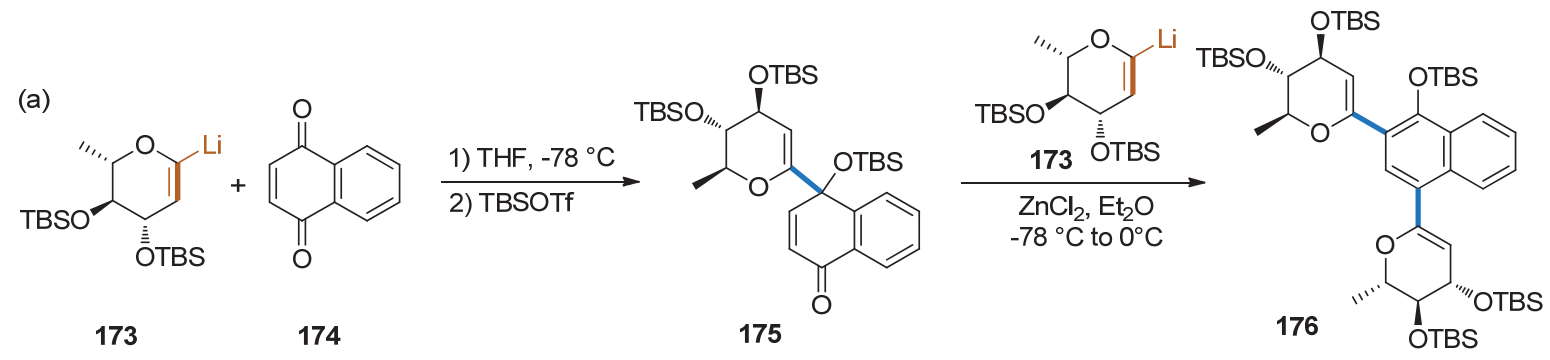<smiles>CC(C)(C)[Si]1(C(C)(C)C)OC[C@H]2OC(Cl)=C[C@H](O[Sb])[C@H]2O1</smiles>

177<smiles>COc1cccc2c1-c1noc3c1C(O)(C2=O)C(C)C[C@H](C)C3</smiles>

178

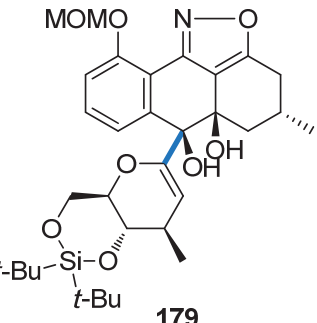

179<smiles>Cc1cc(O)c2c(c1)C(O)([C@H]1O[C@H](CO)[C@@H](O)[C@H](C)[C@H]1O)c1cccc(O)c1C2=O</smiles>

图式 44 烯基锂作为偶联试剂

Scheme 44 Alkenyl lithium as the coupling substrate

(a)

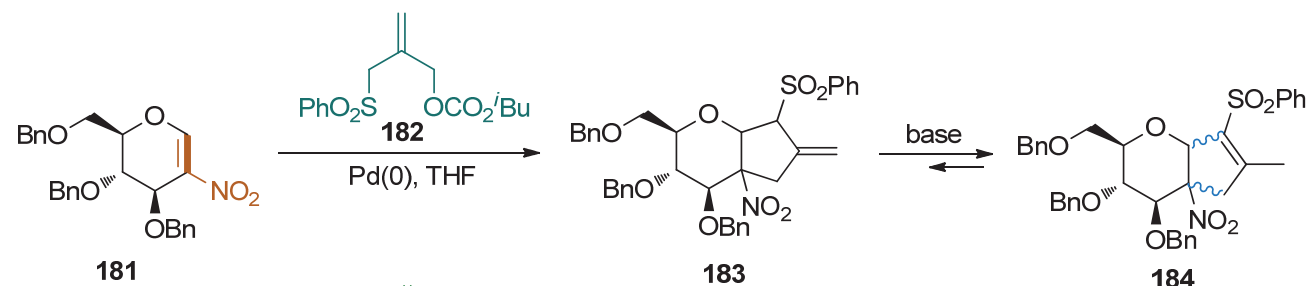

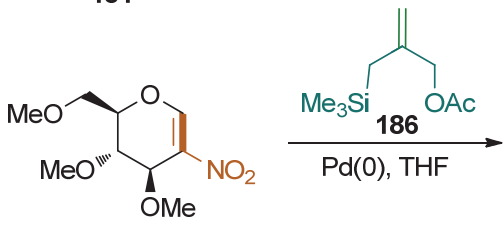

185<smiles>C=C1C[C@H]2O[C@H](COC)[C@@H](OC)[C@H](OC)[C@]2([N+](=O)[O-])[C@@H]1OC</smiles>

187
188

(b)

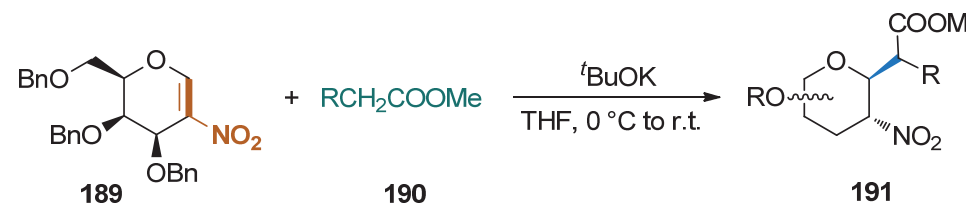<smiles>COC(=O)[C@H]1O[C@H](COCc2ccccc2)[C@@H](O)[C@H](O)[C@H]1[N+](=O)[O-]</smiles>

191a, $74 \%$<smiles>COC(=O)[C@H]1O[C@H](COCc2ccccc2)[C@@H](O)[C@H](O)[C@H]1[N+](=O)[O-]</smiles>

191b, $80 \%$<smiles>COC(=O)[C@H]1O[C@H](COCc2ccccc2)[C@@H](O)[C@H](O)[C@H]1[N+](=O)[O-]</smiles>

191c, $68 \%$

(c)

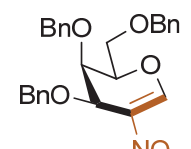

$189 \mathrm{NO}_{2}$<smiles>C=CCCCCCC</smiles>

192 $\underset{82 \%}{\stackrel{\mathrm{THF},-60}{\circ} \mathrm{C} \text { to r.t. }}$

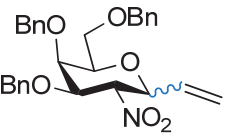

193

图式 45 亲核试剂参与的 Michael 加成反应

Scheme 45 Michael addition of nucleophiles 
(a)

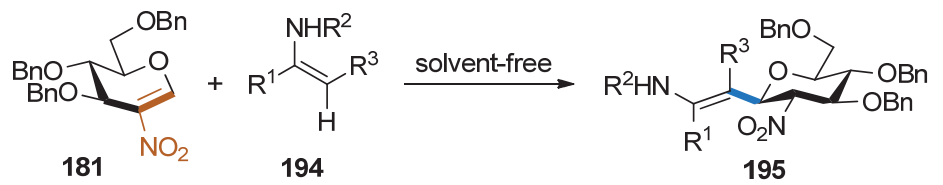

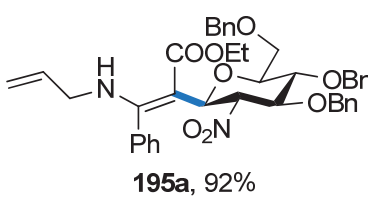

195a, 92\%

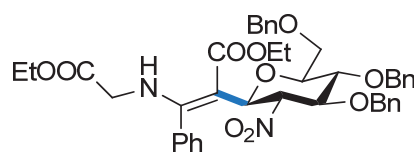

195b, $91 \%$

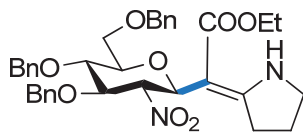

195c, $96 \%, \beta / \alpha=98: 2$

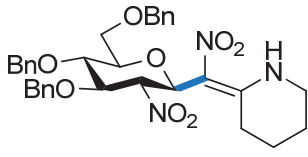

195d, $79 \%$<smiles>[R]C(=O)C1=C([18O])C([R20])[C@@H]([R6])[C@@H]([2H])O1</smiles>

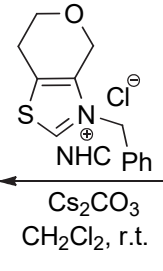<smiles>[R20][C@@H]1OC=C([N+](=O)[O-])[C@@H]([R20])[C@H]1[R20]</smiles>

196
$+{ }_{197}^{\stackrel{\mathrm{O}}{\mathrm{N}}} \mathrm{R}^{1} \frac{\mathrm{NHC}, \text { DIPEA }}{\mathrm{CH}_{2} \mathrm{Cl}_{2} \text {, r.t. }}$

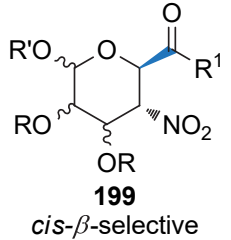

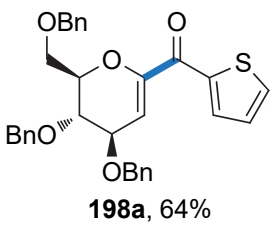

198a, $64 \%$<smiles>O=C(C1=C[C@H](O)[C@H](O)[C@H](COCc2ccccc2)O1)c1ccccn1</smiles>

198b, $82 \%$<smiles>C[C@@H]1OC(C(=O)c2ccccn2)=C[C@@H]([18OH])[C@@H]1O[13CH3]</smiles>

198c, $83 \%$

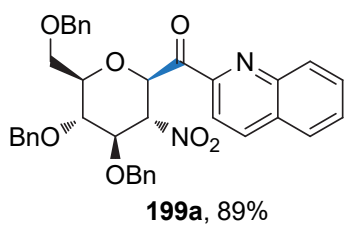<smiles>COC[C@H]1O[C@H](C(=O)c2ccccn2)[C@H]([N+](=O)[O-])[C@@H](OC)[C@H]1O</smiles>

199b, $78 \%$

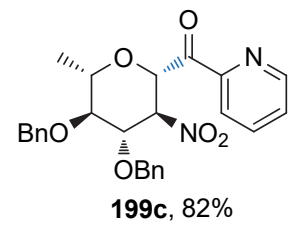

(c)

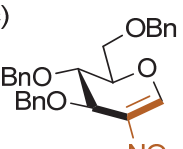
$181 \mathrm{NO}_{2}$

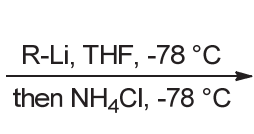
then $\mathrm{NH}_{4} \mathrm{Cl},-78^{\circ} \mathrm{C}$ 200

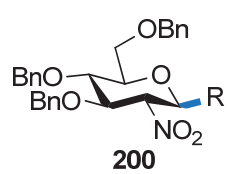

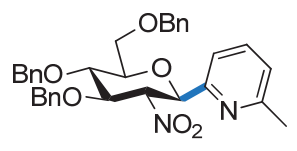

200a, $45 \%$

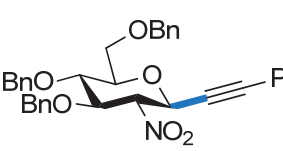

200b, $51 \%$

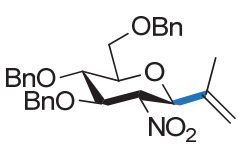

200c, $65 \%$

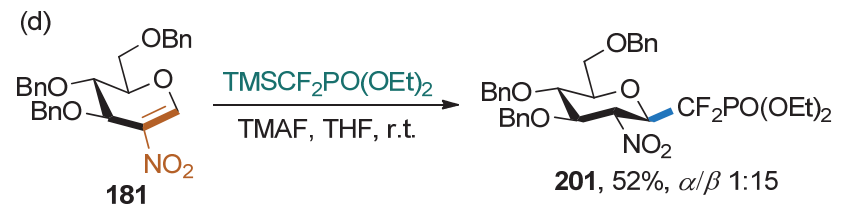<smiles>COCC1(COC)COC(OC)C2COCC1C2[N+](=O)[O-]</smiles>

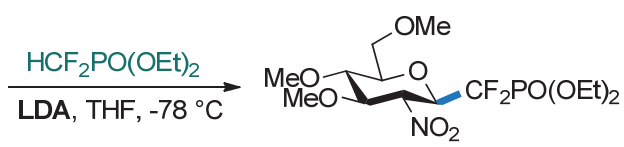

202, 86\%, $d r$ 86:3:11

图式 46 其它亲核试剂的 Michael 加成反应

Scheme 46 Michael addition of other nucleophilic reagents

(a)

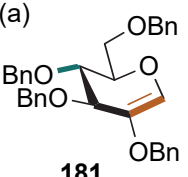

181<smiles>O=C(O)CC1(OCc2ccccc2)COC(=O)C1OCc1ccccc1</smiles>

(b)

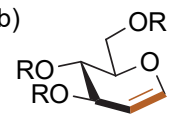

$85, R=B n$ 206, $\mathrm{R}=\mathrm{Me}$

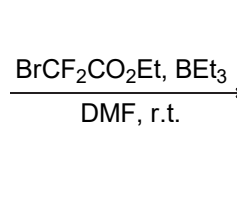

$\underset{\mathrm{DMF}, \text { r.t. }}{\stackrel{\mathrm{BrCF}_{2} \mathrm{CO}_{2} \mathrm{Et}_{3} \mathrm{BEt}_{3}}{\longrightarrow}}$

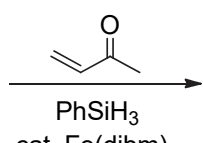
cat. $\mathrm{Fe}(\mathrm{dibm})_{3}$

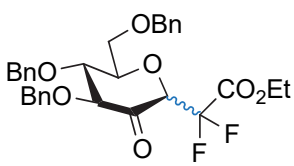

203, $51 \%, \alpha / \beta=3: 1$ $\mathrm{BnO}, \mathrm{OBn}$
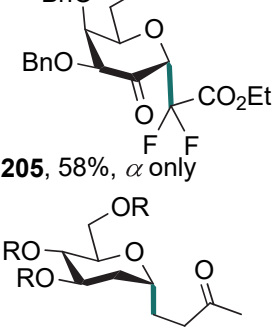

207, $\mathrm{R}=\mathrm{Bn}, 68 \%$ 208, $\mathrm{R}=\mathrm{Me}, 64 \%$
$205,58 \%, \alpha$ only

图式 47 自由基反应合成碳苷

Scheme 47 Synthesis of $C$-glycosides by free radical reactions
(Scheme 47b).

\section{6 总结与展望}

碳苷具有良好的生物活性和成药性，一直是糖化学 家和药物化学家研究的热点, 其独特的 $\mathrm{C}-\mathrm{C}$ 糖苷键是 化学合成的一个挑战. 随着有机化学的飞速发展, 基于 烯烃的 $\mathrm{C}-\mathrm{C}$ 键生成方法也被逐渐引入到碳苷合成. 本 文从 Ferrier I 型碳苷化反应、Heck 偶联型碳苷化反应、 1-取代糖烯的过渡金属催化偶联碳苷化反应、2-取代糖 烯的 Michael 加成型和自由基加成型碳苷化反应等方面 系统地总结了基于糖烯的碳苷合成方法和策略. 从糖烯 出发制备碳苷已成为碳苷合成最有效的策略之一，极大 地加速了碳苷天然产物及类似物的生物活性研究. 尽管 该研究领域已取得较大进展，但仍存在进一步研究和发 
展的空间. 首先, 碳苷通常有两种构型, 但现有大部分 方法只能得到混合物或少部分方法能得到某单一构型 的碳苷, 能通过配体或简单条件改变而实现立体选择性 调控的体系仍然很少, 目前仅限于 3-酮-2-脱氧芳基碳 苷以及 2-吡啶苯基碳苷的合成等极少数例子. 因此发展 立体可控的碳苷合成方法是该领域未来发展的主要方 向之一. 其次, 利用当前方法合成的多为非天然碳苷结 构, 要得到天然碳苷结构通常需要进行多步化学转化; 大部分方法只适用于简单底物, 对于更加复杂的天然苷 元的引入缺乏有效的途径. 随着有机化学的发展和碳苷 化学研究的深入, 相信未来会发展出更加实用和好用的 合成新方法, 并被成功运用于碳苷天然产物合成和新药 研发领域.

\section{References}

[1] Štambaský, J.; Hocek, M.; Kočovský, P. Chem. Rev. 2009, 109, 6729.

[2] Cao, X.; Tian, Y.; Zhang, T.; Li, X.; Ito, Y. J. Chromatogr. A 1999, 855,709 .

[3] (a) Funahashi, Y.; Kawamura, N.; Ishimaru, T. JP 08231551, 1996. (b) Funahashi, Y.; Kawamura, N.; Ishimaru, T. JP 08231552, 1996.

[4] Franck, R. W. Angew. Chem., Int. Ed. 2004, 43, 3818.

[5] (a) Kitamura, K.; Ando, Y.; Matsumoto, T.; Suzuki, K. Chem. Rev. 2018, 118, 1495.

(b) Yang, Y.; Yu, B. Chem. Rev. 2017, 117, 12281.

[6] (a) Gómez, A. M.; Lobo, F.; Uriel, C.; López, J. C. Eur. J. Org. Chem. 2013, 7221.

(b) Vieira, A. S.; Fiorante, P. F.; Hough, T. L. S.; Ferreira, F. P.; Ludtke, D. S.; Stefani, H. A. Org. Lett. 2008, 10, 5215.

(c) Huang, N.; Liao, H.; Yao, H.; Xie, T.; Zhang, S.; Zou, K.; Liu, X. W. Org. Lett. 2018, 20, 16.

[7] (a) Takhi, M.; Rahman, A. H. A.; Schmidt, R. R. Tetrahedron Lett. 2001, 42, 4053.

(b) Anjaiah, S.; Chandrasekhar, S.; Grée, R. J. Mol. Catal. A: Chem. 2004, 214, 133 .

[8] (a) Saeeng, R.; Sirion, U.; Sahakitpichan, P.; Isobe, M. Tetrahedron Lett. 2003, 44, 6211 .

(b) Yadav, J. S.; Reddy, B. V. S.; Rao, C. V.; Chand, P. K.; Prasad, A. R. Synlett 2001, 1638.

[9] Das, S. K.; Reddy, K. A.; Abbineni, C.; Roy, J.; Rao, K. V. L. N.; Sachwani, R. H.; Iqbal, J. Tetrahedron Lett. 2003, 44, 4507.

[10] Ansari, A. A.; Reddy, Y. S.; Vankar, Y. D. Beilstein J. Org. Chem. 2014, 10,300

[11] Steinhuebel, D. P.; Fleming, J. J.; Bois, J. D. J. Org. Lett. 2002, 4, 293.

[12] Tatina, M. B.; Kusunuru, A. K.; Yousuf, S. K.; Mukherjee, D. Org Biomol. Chem. 2014, 12, 7900.

[13] (a) Di Bussolo, V.; Caselli, M.; Pineschi, M.; Crotti, P. Org. Lett. 2003, 5,2173

(b) Bussolo, V. D.; Caselli, M.; Romano, M. R.; Pineschi, M.; Crotti, P. J. Org. Chem. 2004, 69, 7383.

[14] Deelertpaiboon, P.; Reutrakul, V.; Jarussophon, S.; Tuchinda, P.; Kuhakarn, C.; Pohmakotr, M. Tetrahedron Lett. 2009, 50, 6233.

[15] Lubin-Germain, N.; Hallonet, A.; Huguenot, F.; Palmier, S.; Uziel, J.; Augé, J. Org. Lett. 2007, 9, 3679.

[16] Vieira, A. S.; Fiorante, P. F.; Hough, T. L.; Ferreira, F. P.; Lüdtke, D. S.; Stefani, H. A. Org. Lett. 2008, 10, 5215.

[17] Kusunuru, A. K.; Tatina, M.; Yousuf, S. K.; Mukherjee, D. Chem Commun. 2013, 49, 10154.

[18] Hosseyni, S.; Smith, C. A.; Shi, X. Org. Lett. 2016, 18, 6336

[19] Devari, S.; Kumar, M.; Deshidi, R.; Rizvi, M.; Shah, B. A. Beilstein
J. Org. Chem. 2014, 10, 2649.

[20] Chen, H.; Luo, X.; Qiu, S.; Sun, W.; Zhang, J. Glycoconjugate J. 2017, 34, 13

[21] Tan, H. Y.; Xiang, S.; Leng, W. L.; Liu, X.-W. RSC Adv. 2014, 4, 34816.

[22] Dash, A. K.; Madhubabu, T.; Yousuf, S. K.; Raina, S.; Mukherjee, D. Carbohydr. Res. 2017, 438, 1

[23] Yadav, J. S.; Reddy, B. V. S.; Rao, K. V.; Saritha Raj, K.; Prasad, A. R.; Kiran Kumar, S.; Kunwar, A. C.; Jayaprakash, P.; Jagannath, B. Angew. Chem. Int. Ed. 2003, 115, 5356 .

[24] Reddy, G. M.; Maheswara Rao, B. U.; Sridhar, P. R. J. Org. Chem. 2016, 81, 2782.

[25] (a) Moineau, C.; Bolitt, V.; Sinou, D. J. Org. Chem. 1998, 63, 582. (b) Bertini, B.; Moineau, C.; Sinou, D.; Gesekus, G.; Vill, V. Eur. J. Org. Chem. 2001, 2001, 375.

(c) Zeng, J.; Ma, J.; Xiang, S.; Cai, S.; Liu, X.-W. Angew. Chem. Int. Ed. 2013, 125, 5238

(d) Bai, Y.; Leng, W. L.; Li, Y.; Liu, X.-W. Chem. Commun. 2014, 50, 13391.

(e) Leng, W.-L.; Liao, H.; Yao, H.; Ang, Z.-E.; Xiang, S.; Liu, X.-W. Org. Lett. 2017, 19, 416.

[26] Dai, Y.; Tian, B.; Chen, H.; Zhang, Q. ACS Catal. 2019, 9, 2909.

[27] Heck, R. F. J. Am. Chem. Soc. 1968, 90, 5518.

[28] Arai, I.; Daves, G. D. J. Org. Chem. 1979, 44, 21.

[29] Farr, R. N.; Outten, R. A.; Cheng, J. C.-Y.; Daves, G. D., Jr. Organometallics 1990, 9, 3151.

[30] Zhang, H.; Daves, G. D., Jr. J. Org. Chem. 1992, 57, 4690.

[31] Li, H.-H.; Ye, X.-S. Org. Biomol. Chem. 2009, 7, 3855.

[32] Lei, M.; Gao, L.; Yang, J.-S. Tetrahedron Lett. 2009, 50, 5135.

[33] Jovanovic, P.; Petkovic, M.; Simic, M.; Jovanovic, M.; Tasic, G.; Crnogorac, M. D.; Zizak, Z.; Savic, V. Eur. J. Org. Chem. 2019, $2019,4701$.

[34] Tao, Y.; Ding, N.; Ren, S.; Li, Y. Tetrahedron Lett. 2013, 54, 6101.

[35] (a) Rammauth, J.; Poulin, O.; Rakhit, S.; Maddaford, S. P. Org. Lett. 2001, 3, 2013.

(b) Ramnauth, J.; Poulin, O.; Bratovanov, S. S.; Rakhit, S.; Maddaford, S. P. Org. Lett. 2001, 3, 2571.

[36] Figuera, N.; Forns, P.; Fernandez, J. C.; Fiol, S.; Fernandez-Forner, D.; Albericia, F. Tetrahedron Lett. 2005, 46, 7271.

[37] Lai, M.; Othman, K. A.; Yao, H.; Wang, Q.; Feng, Y.; Huang, N.; Liu, M.; Zou, K. Org. Lett. 2020, 22, 1144.

[38] Xiong, D.-C.; Zhang, L.-H.; Ye, X.-S. Org. Lett. 2009, 11, 1709.

[39] Mabit, T.; Siard, A.; Legros, F.; Guillarme, S.; Martel, A.; Lebreton, J.; Carreaux, F.; Dujardin, G.; Collet, S. Chem.-Eur. J. 2018, 24, 14069.

[40] Yoshikawa, Y.; Ishibashi, A.; Murai, K.; Kaneda, Y.; Nimura, K.; Arisawa, M. Tetrahedron Lett. 2019, 60, 151313.

[41] Liu, C.-F.; Xiong, D.-C.; Ye, X.-S. J. Org. Chem. 2014, 79, 4676.

[42] Kusunuru, A. K.; Jaladanki, C. K.; Tatina, M. B.; Bharatam, P. V.; Mukherjee, D. Org. Lett. 2015, 17, 3742.

[43] Bai, Y.; Kim, L. M. H.; Liao, H.; Liu, X.-W. J. Org. Chem. 2013, $78,8821$.

[44] (a) Tang, S.; Zheng, Q.; Xiong, D.-C.; Jiang, S.; Li, Q.; Ye, X.-S. Org. Lett. 2018, 20, 3079.

(b) Xiong, D.-C.; Gao, C.; Li, W.-M.; Wang, Y.; Li, Q.; Ye, X.-S. Org. Chem. Front. 2014, 1, 798.

(c) Liu, M.; Li, B.-H.; Li, T.; Liu, M.; Xiong, D.-C.; Ye, X.-S. Org. Biomol. Chem. 2020, 18, 3043 .

(d) Zheng, Q.; Tang, S.; Xiong, D.-C.; Li, Q.; Ye, X.-S. J. Org. Chem. 2020, 85, 9339.

[45] (a) Singh, A. K.; Kandasamy, J. Org. Biomol. Chem. 2018, 16, 5107.

(b) Singh, A. K.; Venkatesh, R.; Kandasamy, J. Synthesis 2019, 51, 4215.

[46] Xiang, S.; Cai, S.; Zeng, S.; Liu, X.-W. Org. Lett. 2011, 13, 4608.

[47] Kusunuru, A. K.; Yousuf, S. K.; Tatina, M.; Mukherjee, D. Eur. J. Org. Chem. 2015, 2015, 459.

[48] Sakamoto K.; Nagai M.; Ebe Y.; Yorimitsu H.; Nishimura k. ACS 
Catal. 2019, 9, 1347.

[49] Kikuchi, T.; Takagi, J.; Isou, H.; Ishiyama, T.; Miyaura, N. Chem. Asian J. 2008, 3, 2082.

[50] Parkan, K.; Pohl, R.; Kotora, M. Chem.-Eur. J. 2014, 20, 4414.

[51] Oroszova, B.; Choutka, J.; Pohl, R.; Parkan, K. Chem.-Eur. J. 2015, 21,7043 .

[52] Gong, L.; Sun, H.-B.; Deng, L.-F.; Zhang, X.; Liu, J.; Yang, S.; Niu, D. J. Am. Chem. Soc. 2019, 141, 7680.

[53] Dubbaka, S. R.; Steunenberg, P.; Vogel, P. Synlett 2004, 1235.

[54] Koo, B.; E. McDonald, F. Org. Lett. 2005, 7, 3621.

[55] Hartung, J.; Wright, B. J. D.; Danishefsky, S. J. Chem.-Eur. J. 2014, 20,8731 .

[56] Koester, D. C.; Kriemen, E.; Werz, D. B. Angew. Chem. Int. Ed. 2013, 52, 2985.

[57] Potuzak, J. S.; Tan, D. S. Tetrahedron Lett. 2004, 45, 1797.

[58] (a) Liu, M.; Niu, Y.-H.; Wu, Y.-F.; Ye, X.-S. Org. Lett. 2016, 18 , 1836.

(b) Wang, H.; Niu, Y.-H.; Zhang, G.; Ye, X.-S. Tetrahedron Lett. 2016, 57, 4544.

[59] Zhang, S.; Niu, Y.-H.; Ye, X.-S. Org. Lett. 2017, 19, 3608.

[60] Liu, Y.; Wang, Y.; Dai, W.; Huang, W.; Li, Y.; Liu, H. Angew. Chem. Int. Ed. 2020, 59, 3491

[61] Wu, J.; Kaplaneris, N.; Ni, S.; Kaltenhauser, F.; Ackermann, L. Chem. Sci. 2020, 11, 6521.

[62] Boucard, V.; Larrieu, K.; Lubingermain, N.; Uziel, J.; Augé, J. Synlett 2003, 1834.

[63] Marjolein, V. D. K.; Eefjan, B.; Pieters, R. Beilstein J. Org. Chem. 2012, 8,732 .

[64] Parker, K. A.; Koh, Y. H. J. Am. Chem. Soc. 1994, 116, 11149.

[65] Yasuhito, K.; Ryo, Y.; Keisuke, S. Angew. Chem. Int. Ed. 2008, $120,1100$.
[66] Holzapfel, C. W.; Merwe, T. L. V. D. Tetrahedron Lett. 1996, 37, 2307.

[67] Pachamuthu, K.; Gupta, A.; Das, J.; Schmidt, R. R.; Vankar, Y. D. Eur. J. Org. Chem. 2002, 1479.

[68] Reddy, B. G.; Vankar, Y. D. Angew. Chem. Int. Ed. 2005, 44, 2001.

[69] Jayakanthan, K.; Vankar, Y. D. Tetrahedron Lett. 2006, 47, 8667.

[70] Zhang, T.; Yu, C.-Y.; Huang, Z.-T.; Jia, Y.-M. Synlett 2010, 2174.

[71] Vedachalam, S.; Shi, M. T.; Hui, P. T.; Cai, S.; Liu, X.-W. Org. Lett. 2012, 14, 174.

[72] (a) Delaunay, S.; Poisson, T.; Jubault, P.; Pannecoucke, X. Eur. J. Org. Chem. 2014, 3341.

(b) Verma, A. K.; Chennaiah, A.; Dubbu, S.; Vankar, Y. D. Carbohydr. Res. 2019, 473, 57

[73] Delaunay, T.; Poisson, T.; Jubault, P.; Pannecoucke, X. J. Fluorine. Chem. 2015, 171, 56.

[74] Bouwman, S.; Orru, R. V. A.; Ruijter, E. Org. Biomol. Chem. 2015, $13,1317$.

[75] (a) Lopez, J. C.; Fraser-Reid, B. J. Am. Chem. Soc. 1989, 111, 3450. (b) Gomez, A. M.; Casillas, M.; Valverde, S.; Lopez, J. C. Tetrahedron: Asymmetry 2001, 12, 2175.

(c) Li, G.; Xiong, D.-C.; Ye, X.-S. Synlett 2001, 2410.

[76] (a) Moreno, B.; Quehen, C.; Rose-Helene, M.; Leclerc, E.; Quirion, J.-C. Org. Lett. 2007, 9, 2477.

(b) Colombel, S.; Van Hijfte, N.; Poisson, T.; Leclerc, E.; Pannecoucke, X. Chem.-Eur. J. 2013, 19, 12778.

[77] (a) Lo, J. C.; Gui, J.; Yabe, Y.; Pan, C.-M.; Baran, P. S. Nature 2014, 516, 343.

(b) Lo, J. C.; Kim, D.; Pan, C.-M.; Edwards, J. T.; Yabe, Y.; Gui, J.; Qin, T.; Gutiérrez, S.; Giacoboni, J.; Smith, M. W.; Holland, P. L.; Baran, P. S. J. Am. Chem. Soc. 2017, 139, 2484. 